Werner Abraham

ANDERS 1.DOC/09-03-99 12:08

abraham@let.rug.nl

\title{
Überlegungen zum Passiv im Deutschen und anderen Sprachen: 'Argumenthypothese' und 'Aspekthypothese'
}

\author{
"A challenge in which a successful outcome is assured isn't a challenge." \\ Jon Krakauer Into the wild. 1996
}

\subsection{Die Passiverscheinung zwischen zwei erklärenden Grundannahmen}

Die Ableitung des Passivs ist typologisch keine einheitlich konfigurierte Konstruktion. In den kontinental-westgermanischen Sprachen und dem Lateinischen setzt sie ein lexikalisch externes Argument (designiertes Subjektargument) voraus, im Englischen, Französischen und Russischen sowohl ein externes wie ein internes Argument (Subjekt und (direktes) Objekt). Gleichwohl sind Passive im Deutschen und Russischen - also quer zu dieser ersten Verbklassifikation - aspektuellen Beschränkungen unterworfen, Passive im Englischen dagegen nicht, jedenfalls auf. der ersten Blick. Sehen wir in diesen Kreis von Sprachen noch historische Stufen hinzu, dann ist auch davon auszugehen, daß Sprachen wie das Deutsche von einer Stufe mit einem paradigmatisch einigermaßen systematisch gefestigten Aspektsystem ohne Passiv - dem Althochdeutschen - zu einer Sprache mit Passiv (und ohne Aspekt) wurde. Wir brauchen gar nicht die gemeinsame indoeuropäische Wurzel zu beschwören, um die folgenden Fragen plausibel erscheinen zu lassen: Was hat Aspekt mit Passiv zu tun? Und: Soferne solche Übergänge tatsächlich vorliegen wie sehen die Schritte von Aspekt zum Genus verbi im einzelnen aus, und wo stehen die Sprachen heute im Vergleich zueinander, also auf einer Art Entwicklungsleiter, mit Vorläufer- gegenüber Nachläuferstufen in der relativen Diachronie von Aspekt zur Passivdiathese?

Es sollen diese Fragen im weiteren unter den versammelnden zwei Hypothesen angesprochen werden: der 'passivischen Argumenthypothese' sowie der 'passivischen Aspekthypothese'. Damit ist gemeint, daß die Passivableitung rein unter Inachtnahme der Argumentstruktur der Ausgangsprädikate abläuft oder aber eben unter aspektuellen Kriterien. Die 'Argumenthypothese' versammelt die in der modernen Syntax bekannten Passivierungskriterien, die ausnahmslos bestimmt sind durch Manipulationen der Argumente des diathetisch abzuleitenden Prädikats. Die 'Aspekthypothese' dagegen betrachtet das verbale Genus als Epiphänomen, das aus einer Aspektsystematik hergeleitet wird. Dazwischen gibt es, so die opinio communis (vgl. etwa Schoorlemmer 1995), Übergänge, vor allem im typologisch synchronen Nebeneinander, aber auch in historischer Longitudinalität. $\mathrm{Ob}$ alle genannten Passivbedingungen in einem Szenario zwischen den beiden Polen unterzubringen sind - etwa die Argumentunterschiede zwischen Deutsch und Englisch und die Erwägung, daß das Deutsche aspektuelle Passiveinschränkungen kennt, das Englische hingegen nicht - , muß vorerst offen bleiben.

Bei der Suche nach Antwortrouten zu den oben gestellten Fragen habe ich mich von der vorerst noch unentschiedenen - Annahme leiten lassen, daß Aspekt bzw. Aktionsart auch im modernen Deutschen eine dem Verbgenus vorgeschaltete Grammatikkategorie ist. Diese Annahme ist vorerst rein methodisch getroffen: Es ist zu prüfen, inwieweit das Deutsche dem Rus- 
sischen ähnelt, zumal ja das Althochdeutsche noch über ein paradigmatisch gesichertes Aspektund Aktionsartsystem, aber noch keine systematische Passivableitung verfügte. Wie, so sollte man angesichts dieses diachronen Bestands fragen, kam es von einem Aspekt- zu einem Genussystem im modernen Deutsch, wenn nicht Aspekt und Passiv nahtlos aneinander anschließen können? Der zweite Beweggrund dafür gerade diese Marschroute zu wählen liegt in der Einsicht, daß die deutsche Sprechsprache auf dem Weg von Aspekt zu Tempus gegenüber dem Schriftdeutschen wieder einen Schritt voraus ist - damit nämlich, daß sein periphrastisches Perfekt reine Imperfekt-(/Aorist-)Bedeutung erlangt hat. Wir werden also in den folgenden inhaltlich geordneten Abschnitten aus diesem Erscheinungsbereich neue Fakten in die Argumentation miteinzubringen haben.

\section{2. 'Argumenthypothese' und 'Aspekthypothese'}

Mit der Linguistik der Passivdiathese liegt etwas Sonderbares insoferne vor, als sein Aussehen und seine Erklärung im Gotischen, Althochdeutschen und Mittelhochdeutschen weitaus klarer ist als im modernen Deutschen. 'Passivsinn' war in dieser Vormoderne des Deutschen auf perfektive Prädikate beschränkt; seine Syntax war im wesentlichen die des Objektprädikats. Passivsinn setzte in dieser Vorperiode des modernen Deutschen somit transitive Verben voraus - was ja für das heutige Deutsch nicht mehr gilt. Der Terminus 'Passivsinn' (im Unterschied zu 'Passivmorphologie') hat angesichts der Verhältnisse in diesen historischen Abschnitten des Deutschen nichts Befremdliches - um wieviel mehr dagegen, wenn man vom heutigen Deutschen oder Englischen spricht, wo man einfach davon ausgeht, 'daß es ein Passiv gibt' und daß ein solches Passiv ein eigenes Verbgenus ist (vgl. die Terminologie voice; voix im Romanischen!) bzw. zu den Verbdiathesen gehört - also in die Nähe solcher Kategorien wie Kausativ-Dekausativ, ReflexivDereflexiv, Transitiv-Detransitiv etc.. Dabei ist eigentlich nichts merkwürdiger, als daß man so einfach von einem 'Passiv' im Deutschen und Englischen spricht - anders als etwa in den slawischen oder skandinavischen Sprachen oder im Latein, wo es ja jeweils eine eigene synthetische Passivmorphologie (jedenfalls in einem Teil der Tempusparadigmen) gibt. Denn: Wie ist der Wandel von der aspektuell geladenen Objektprädikation in den Perioden vor dem Frühneuhochdeutschen zum verbalen Passivgenus im Nhd. eigentlich zu verstehen: Kam da einfach so eine neue Kategorie im Verbsystem dazu, soz. aus heiterem Himmel?

Sprechen wir über diese unerklärte diachrone Erscheinung in der Folge der Einfachheit halber vom 'Passivrätsel'. Das Passivrätsel bezeichnet die unerklärte Erscheinung, daß im Nhd. plötzlich ein 'Verbgenus' auftaucht, das es vorher nicht gegeben hat. Man beachte, daß zur Erklärlichkeit um nichts mehr geleistet wird, wenn man sich auf die Diathesenterminologie - also die Valenzfrage - einläßt. Denn: Passivierung besteht ja nicht einfach darin, daß die 'Passivform' die Agensstelle einbüßt - das tut sie ja nun gerade nicht - und schon gar nicht, daß das ursprüngliche Subjekt der Aktivdiathese in der 'Passivdiathese' den Satzgliedstatus mit dem ursprünglichen direkten Objekt tauscht - etwa was 'Inversion' eigentlich suggerieren würde. Gewiß: die verbalen Argumente werden in ihrer syntaktischen Form in einer gewissen Weise invertiert - aber auch nur in einem sehr spezifischen Inversionsverständnis, keinesfalls so wie man geben und erhalten in einer Inversionsbeziehung inbezug auf ihre beiden thematischen Rollen GOALIZIEL bzw. AGENS sehen kann.

Das Passivrätsel wird noch gravierender, wenn man die Erlernbarkeit des Passivs mit in Betracht zieht. Gleichgültig wie man der L1-Lernfrage nachgeht - d.h. ob man davon ausgeht, daß die Passivsyntax nach Maßgabe der erlernten Lexeme zustandekommt oder daß das Erlernen 
der syntaktischen Passivoperationen Bedingung dafür sind, daß lexikalische Elemente mit ihren Passivierbarkeiten gelernt werden -: es ist völlig unklar, wie das Kind erkennt, daß das 2. Partizip aktiv-perfektische oder passivische Bedeutung hat. Dies kann auf keinen Fall unter der lexikalischen Lernhypothese geschehen. Daß dies aber auch unter der syntaktischen Lernhypothese zu Aporien führt, zeigen die folgenden drei Schritte. Wir gehen von der prinzipiellen Homonymie des Partizipialmorphems, PM, aus, etwa ndl. begonnen.

(1)a begonnen: aktivisch, also begonnen (hebben) oder passivisch, begonnen (worden/(geworden) zijn)?

b Aux-Selektion kann zur Entscheidung der Frage in (1a) nicht beitragen, denn das von begonnen selegierte zijn löst sowohl aktivische wie auch passivische Bedeutung aus: vgl. das passivische het is begonnen (geworden) bzw. aktivisch-perfektisches wij zijn ' $t$ begonnen. Zu einer nicht unbeträchtlichen Reihe solcher AuX-Doppelfunktionen vgl, allgemein die Grammatikliteratur des Deutschen.

c Selbst die Annahme, daß begonnen nach dem Kriterium einer Objektselektion entweder Passivpartizip oder aber Perfektpartizip ist, führt in eine Aporie und zwar deswegen, weil beginnen einwertig und zweiwertig (zweiwertig mit DO-Ellipse oder einwertig?) yerwendet werden kann.

Wie man es also dreht und wendet, die L1-Erlernbarkeit scheint zumindest bei einer Reihe von Lexemen, die sich wie ndl. beginnen verhalten, ausgeschlossen zu sein; sie wird bei diesen Lexemen auf jeden Fall lexikalisch verlaufen müssen, weil die Syntaxentscheidung nicht möglich ist - was ja nun auch nicht gerade zur Stabilisierung einer zu erlernenden Passivsyntax beiträgt.

Schließlich ist die Annahme einer systematisch zu entscheidenden (komputationellen!, algorithmisch verlaufenden!) Passivsyntax auch angesichts der L2-Erlernbarkeit nicht plausibler: haben doch eine Reihe von Sprachen überhaupt kein Passiv im modern-westgermanischen Sinne.

\subsection{Konstruktionskomponenten des verbalen Passivs, etwa im Deutschen oder Niederländischen oder Englischen:}

Man vgl. im folgenden das, was in der Literatur ohne Abstriche aufgrund schulischer Voreingenommenheit als unverzichtbare Konstruktionskomponenten des Passivs gehandelt werden (zu Untenstehendem vgl. Shibatani (1985), der sich freilich nicht zum Deutschen äußerte).

(2)a Das Perfektpartizipmorphem (PM) selegiert werden/worden/been oder sein/zijn/be. Ent scheidungskriterium: AUX-SELEKTION.

b Satzgliedvorrückung des direkten Objekts ('Kasuszuweisungsprinzip für NP'); Verlust der Akkusativrektion ('biimplikatives Kasuszuweisungsprinzip'). Entscheidungskriterium: KASUSFILTER - '*NP OHNE KASUSZUWEISUNG'.

c das ursprüngliche (lexikalisch designierte) externe Argument wird rückversetzt zu einem Präpositionsglied (komputationelles syntaktisches Prinzip?); es bleibt in jedem Falle im plizit. Entscheidungskriterium: 'BINDUNG DER AGENS-ROLLE AN DAS PASSIVMORPHEM AUF LF' (was immer das genau heißen möge). 
Es ist alleine diese Liste an Verbgenuseigenschaften höchst metaphorisch, für einen Algorithmus höchst undurchsichtig und komputationell kaum verwendbar. Was aber noch schlimmer ist: es sind zum einen die Zusammenhänge zwischen (a), (b) und $\mathbb{C}$ in (2) nicht klar, würde man sich doch wünschen, daß solche Passivmerkmale nicht ohne inneren Zusammenhang separat postuliert werden müßten; und zweitens ist ebenso unklar, was $(2 \mathrm{a}-\mathrm{c})$ mit der Objektprädikation des Althochdeutschen zu tun hat, wie es aus ihm entstanden ist und was diese Liste mit jenen. Erscheinungen zu tun hat, die in nichtpassivierenden Sprachen durchaus ähnliche Textverknüpfbarkeiten zustandebringen (Problem der Übersetzbarkeit bei fehlenden kategorialen Äquivalenzen). Wie, so muß man sich fragen, löst der ungarische oder finnische Deutsch- oder Niederländischlerner die essentiellen Komponenten der syntaktischen Passivanalyse aus dem Bild des sich ihm bietenden Verbgenuskomplexes im Deutschen bzw. Niederländischen - nicht nur wo seine eigene Sprache überhaupt kein Verbgenusäquivalent besitzt, sondern wo die deutschen oder niederländischen Erscheinungen noch dazu ein so unentscheidbares Bild liefern wie oben?

Es sind solche Argumentszenarien, die zu einer solchen Alternative führen, wie sie im folgenden dargestellt wird und die in der Behauptung gipfelt, daß dasjenige, was man Passiv nennt, nur 'Passivsinn' hergibt, aber keinesfalls ein eigene formal-paradigmatische Morphologie einer Passivdenotation besitzt. Wir werden sagen, daß die in (2a-c) dargelegten Passivkonstruktionskomponenten Epiphänomeme neben etwas ganz anderem sind. Dabei hat die Frage, welche Grundbedeutung das PM hat - aktivische Perfektbedeutung oder passivische Präsensbedeutung in der ganzen Diskussion Hauptstatus: Wir werden sagen, daß das PM immer nur eine Bedeutung, nämlich aktivisch-perfektische hat.

Die Frage wird schließlich sein, was wir mit solchen Beschreibungskomponenten gewinnen - vor allem gegenüber der herkömmlichen Beschreibung, etwa nach (2a-c). Die Frage, wie die moderne Linguistik eine evident falsche Spur verfolgen konnte, ist zweifellos auch dem Vorbildcharakter jener Sprachen anzulasten, die ein unzweifelbares, weil synthetisches Passiv haben; dazu gehört das Lateinische. Was gewinnen wir aber nun mit der Annahme, daß die Grundbedeutung des 2. Partizips immer die des PPA ist - nachdem wir erörtert haben, was wir an Plausibilität und Gesamtverständnis nicht erreichen bzw. verlieren?

Betrachten wir die Plausibilitätsfrage zuerst diachron. Nach dem Bild im Althochdeutschen (und ebenso im Gotischen und Mittelhochdeutschen, bei letzterem freilich mit abnehmender Gültigkeit; vgl. Abraham 1987, 1993) ergibt sich ein minimaler syntaktischer Unterschied, ablesbar aus (1). [Der deutsche Satz ist dem bekannten notkerschen Satz nachgebildet]..

(3) Er hat den Baum gepflanzt

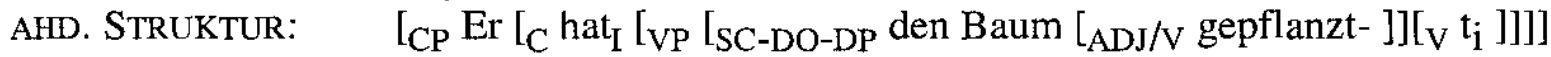
habêt boum gipflanzotan

= SPÄTLAT. STR.: habet arborem plantatum

NHD. STRUKTUR: [ ${ }_{\mathrm{CP}} \operatorname{Er}_{\mathrm{C}_{\mathrm{C}}}$ hat $_{\mathrm{I}}$ [VP $_{\mathrm{V}}$ [DO-DP $_{\mathrm{D}}$ den Baum] [V gepflanzt $\left.\left.\left.\left.\mathrm{t}_{\mathrm{i}}\right]\right]\right]\right]$ ${ }_{\mathrm{CP}} \operatorname{Er}\left[{ }_{\mathrm{C}}\right.$ bekam ${ }_{\mathrm{I}}\left[\mathrm{VP}\left[\mathrm{SC}-\mathrm{DO}-\mathrm{DP}\right.\right.$ den Baum [ $\mathrm{ADJ}_{\mathrm{V}}$ schon gepflanzt $\left.\left.\left.\left.]\right]\left[{ }_{\mathrm{V}} \mathrm{t}_{\mathrm{i}}\right]\right]\right]\right]$ $\cong$ Er bekam den Baum (als) gepflanzten

Aus (3) ist ein direkter, d.h. total unenigmatischer Zusammenhang zwischen Objektprädikation zum Ahd./Mhd. mit SC-Syntax und Vollverb haben mit postponiertem, nach nominalem Genus, Kasus sowie Numerus flektiertem partizipialem Attribut sowie dem modernen Perfektpartizip mit haben als Hilfsverb und ohne Konguenz mit dem DO ersichtlich. Zudem besteht formale Ho- 
monymie und der intendierte semantische Unterschied zwischen dem ahd. und der nhd. Fassung mit bekommen, wo auch im Nhd. Objektprädikation mit haben als Vollverb und dem 2. Partizip von gepflanzt als postponiertes Attribut zum DO den Baum vorliegt. Der Gewinn der Erklärung des deutschen Passivs aus Aspekt- und Aktionsartbedingungen würde darin liegen, daß dieser Übergang auch syntaktisch plausibler gemacht würde.

Was gewinnen wir unter synchroner Betrachtung mit der Annahme 'der Passivsinn des 2. Partizips ist aus der Zustandsbedeutung des PPM abgeleitet; Passivität kann formal nicht direkt repräsentiert werden'? Zum einen entsteht erst gar nicht die Frage nach dem Genuswechsel beim Verb, also dem Komponentenwechsel zwischen Aktiv und Passiv nach (2ac). Denn: was ist denn 'Passiv' anderes als ein Verbmorphemwechsel derart, daß \{haben/ sein $\}_{\text {aktiv }} \Rightarrow\{\text { werden/ ein }\}_{\text {passiv }}$ wechselt, daß ferner die thematische Struktur (Valenz) bei Erhalt der semantischen Valenz syntaktisch reduziert wird? Daß immer wieder hervorgehoben wird, daß das Passiv mit seinem vorgerückten Subjekt ein neues Textthema produziert (siehe die prominente Stelle, die dieses Argument auch bei Shibatani 1985 einnimmt!), hat ja mit der Sạtzgrammatik nichts zu tun und könnte überdies jederzeit auch über DO-Topikalisierung erreicht werden - wir lassen dieses Argument völlig links liegen). D.h. es liegt gar nicht an einer 'passivischen' Verbmorphologie wie etwa im lateinischen Präsens, Imperfekt und Futur (Indikativ wie Konjunktiv, die ausnahmslos synthetisch gebildet sind), dem russischen sja-Passiv oder dem skandinavischen $s$-Passiv. Die Frage, wie das 'Passiv' dann zustandekommt, wollen wir dann so beantworten: das Partizipialmorphem des Perfekts hat lexikalisch designierte, festgelegte thematische und aspektuelle Eigenschaften, die die Nichtagentivität der aktuellen Passivinterpretation bei gleichzeitiger Agensimplikation in der aspektuell begründeten Präsuppositionskomponente ableitbar macht. Dies macht die weitere Frage nach einer eventuellen Passivbedeutung des 2 . Partizips überflüssig. Die Frage, ob das 2. Partizip prinzipiell aktivische oder passivische Bedeutung hat, stellt sich erst gar nicht. Die reine Zustandsbedeutung des PPM erlaubt ja ohne weitere Bedingung keine Agensvalenz. Das Präteritalpartizip hat aufgrund der Zustandsbedeutung prinzipiell unagentischen Adjektivstatus.

Gehen wir zur 2. Definitionskomponente nach (2b) oben: das designierte Agens wird zur Präpositionalkonstituente 'degradiert'. Was daran komputationell interessant ist, hat damit zu tun, daß eine solchermaßen erreichte Verbdiathese nicht weiteren syntaktischen Diathesewechseln (Valenz- oder Argumentreduktionen, aber eben auch wieder nicht semantisch!) aussetzbar ist. Aber sonst liegt hiermit reinste, komputationell unbrauchbare Metaphorik vor - allemal wohl entlehnt der Relations- bzw. Arc-Pair-Grammatik mit deren suggestiven Schaubildern. Erklärenden Status hat dagegen, wenn man sagt, daß ein in der sein/werden-Selektion zum (Eigenschafts-)Adjektiv (d.h. von $[+\mathrm{V},-\mathrm{N}]$ zu $[+\mathrm{V},+\mathrm{N}]$ ) umkategorisiertes 2. Partizip kann kein als Subjekt designiertes Agens haben. Die Frage, ob eine Kategorie ein bestimmtes thematisches Subjektargument tragen kann, beantwortet aber noch nicht die Frage, ob dieses Agens nicht in Abhängigkeit von einer anderen Bedeutungskomponente des 2. Partizips impliziert ist und als anderes Satzglied bzw. in anderem syntaktischem Argumentstatus auftauchen kann. Dazu bietet sich, wenn das Partizipadjektiv als Resultat eines vorausgehenden Entwicklungsereignisses interpretierbar ist, eine entsprechende semantische Rolle an: eben HERKUNFT (SOURCE) ausgedruckt durch dt. von (bei Personsurhebern), durch (bei Sachurhebern); av; par oder durch ein Begleitumsytands-P: para; by. Man vergleiche die lexikalisch-syntaktische Parallele zwischen dem passivischen von/durch-Agens und der rein lexikalischen Diathese in ( $A G$ geb-GOAL/REZ $) \Rightarrow(G O A L / R E Z$ erhalt- [SOURCE/HERKUNFT von AG]). Bereits die Tatsache, daß man in der Wahl dieses Herkunftslexems nicht grammatisch gebunden, sondern eine gewisse 
lexikalisch-semantische Freiheit hat, weist auf die lexikalisch zu erklärende Systematik hin sowie darauf, daß hiermit keine bloß syntaktisch zu deutende Erscheinung vorliegt.

Schließlich spiegelt sich die spezifische Agensmorphologie zwischen Aktiv und Passiv auch in den Ergativsprachen und zwar insofern, als die Ergativmorphologie auf flektivische Präpositionsinkorporation zurückgeht (Mahajan 1994; vgl. auch Abraham 1993). D.h. der Agensergativ - der Nichtpivotkasus eines Kasusergativsystems - war ursprünglich der herkunftsandeutende Kasus, wie es der Genitiv bzw. Ablativ im Lateinischen ist, während der Absolutiv als Pivotsatzglied in der Diathesenverwandtschaft zwischem zweiwertigem und einwertigem Verb die unmarkierte Verbform trägt.

Es gibt im Westgermanischen und besonders im Deutschen unmittelbar einsichtige Hinweise auf die Passivherleitung im Sinne der Aspekthypothese. Der spezifische Aktiv-Passivwechsel bei perfektivem bzw. imperfektivem Verblexem, der allein vom Verbgenus her unerklärlich ist, findet dann ohne jegliche Zusatzannahme eine Lösung. Ich komme darauf schwerpunktmäßig noch zurück. Man vgl. dazu vorerst (4).

\section{(4)a der Wagen wird geschoben der Wagen $*$ ist geschoben \\ b der * geschobene Wagen der * getauchte Schwimmer}

\author{
PERFEKTIVVERB \\ der Wagen wird hineingeschoben \\ der Wagen ist hineingeschoben \\ der hineingeschobene Wagen \\ der untergetauchte Schwimmer
}

Im übrigen ist die Auxiliarselektion nur deshalb lexikalisch ableitbar, weil sie in der lexikalisch festgelegten Aktionsart des Einzelverblexems, nämlich in der Opposition 'telisch $\neq$ nichttelisch' begründet ist. Vgl. dazu nochmals das sein/zijn-Verb in (1) in beiden Diathesen wie im ndl. $H i j(A G)$ is $i e t s(T H)$ begonnen/verloren/vergeten als Aktiv-Perfektiv sowie Het(TH) is begonnen/verloren/vergeten als Zustandspassiv-Perfektiv. Man vgl. dazu die Fortbewegungs(in)transitiva im Deutschen wie (nach Athen) gelaufen/geschwommen sein $\neq$ (den ganzen Tag) gelaufen haben bzw. das Buch durchgegangen sein . Aber natürlich: nicht die Auxiliarwahl alleine ist hier in allen Fällen diagnostisch verläßlich. D.h. als Signal für den Diathesewechsel kann nicht das Hilfsverb gelten (etwa sein gegenüber haben - was in den herkömmlichen Grammatiken des Deuts0chen herumgeistert!), sondern einzig und alleine die lexem-individuelle semantisch-aspektuelle Ereignisstruktur des Verbs. Daß jedoch ohne Wechsel zwischen Aktiv und (Zustands-)Passiv einerseits das Hilfsverb identisch bleibt wie in ... is [...] begonnen in (1) oben und gleichzeitig das Subjekt wie bei Passivierung von AGENS zu PATIENS/THEMA übergeht, beweist nachhaltig, daß die Aktiv-Passiv-Diathese aspektuell zumindest mitbegründet ist. Schließlich ist auch die ndl. Tilgung des geworden-Partizips direktes Zeugnis für den 'Perfekteffekt': es wird die Inchoativphase beim Perfektivpartizip des biphasischen Prädikats durch PA+ zijn/ sein einfach impliziert. Damit wird die Unterscheidung zwischen Vorgangspassiv und Zustandspassiv - vgl. de veldslag is geslagen (??/* geworden) "die Schlacht ist geschlagen (worden)" - einfach suspendiert - sie erweist sich für das Ndl. als unnötiger Luxus. Dies spiegelt sich in den sein-Formen der Frühstadien des Deutschen (Behaghel 1924: §647-650).

Orientieren wir uns also zuerst an den lexikalischen und syntaktischen passivähnlichen Formen im Germanischen und Russischen. 
3. Die formalen Unterschiede zwischen analytischer und synthetischer Passivform - Deutsch, Schwedisch, Russisch

\subsection{Persönliches und unpersönliches Vorgangspassiv}

Im Russischen ist das periphrastische Passiv zum Unterschied vom synthetischen, diachron auf Reflexivierung zurückgehendenm Passiv rein aspektuell bzw. aktionsarttechnisch begründet. Dies war auch der Zustand des Gotischen und Althochdeutschen bis ins Mhd. hinein. Natürlich sind weder die Hilfsverben noch die Reflexivierung identisch. Man hat zu unterscheiden: unter den Hilfsverben solche mit werden-Bedeutung (schwed. bli) und sein-Bedeutung (russ. byf); unter den Reflexivmorphemen freie $(s i c h ; s i g)$ und gebundene $(-s ;-s j a)$. Nur das freie Reflexiv scheint eine eigene thematische Rolle übernehmen zu können - muß dies aber nicht tun, wie das deutsche Medium zeigt (wo es das AGENS bindet(?)). Und: In einem klar perfektivisch kontrollierten Szenario wie im Russischen gliedert sich keine Möglichkeit eines 'unpersönlichen Passivs' aus - wir werden unten darstellen, daß sich dies aus der Aspektkonfiguration ergibt. Sehen nun synthetische Passive in ihrer grammatischen Verteilung anders aus als periphrastische? Vgl. Abb. 1 .

Abb. 1: unpersönliches Passiv zwischen Reflexiv und periphrastischer Komposition

(auf der Grundlage von Andersson 1998 weitergebildet)

\begin{tabular}{|c|c|c|c|}
\hline & PASSIVFORM & VORGANGSPASSIV & $\begin{array}{l}\text { UNPERSÖNLICHES } \\
\text { 'PASSIV' }\end{array}$ \\
\hline \multirow[t]{2}{*}{ Deutsch } & $\begin{array}{l}\text { werden+2.Par- } \\
\text { tizip }\end{array}$ & Das Haus wurde gebaut & Es wurde getanzt \\
\hline & $\begin{array}{l}\text { passivähnl. 'Medium': } \\
\text { ohne Aspektdiffe- } \\
\text { renzierung }\end{array}$ & $\begin{array}{l}\text { Ein solches Haus baut } \\
\text { (es!) sich nicht } *(\text { leicht }) \\
\text { fertig }\end{array}$ & $\begin{array}{l}\text { Polkas tanzt es/tanzen } \\
\text { sich } *(\text { leicht }) ; \text { Es tanzt } \\
\text { sich gerne Polka }\end{array}$ \\
\hline \multirow[t]{2}{*}{ Schwedisch } & blit+2. Partizip & Huset blev byggt & $O\left({ }^{*}\right.$ Det blev danset $)$ \\
\hline & Verbstamm+s & Huset byggdes & Det dansades \\
\hline \multirow[t]{3}{*}{ Russisch } & $\begin{array}{l}\text { imperfekt.V: } \\
\text { Stamm+sja }\end{array}$ & Dom stroilsja & $\bar{O}$ \\
\hline & $\begin{array}{l}\text { perf.V: } b y l^{\prime}+2 . \text { Par- } \\
\text { tizip }\end{array}$ & Dom byl postroen & 0 \\
\hline & Infinitiv & (po)stroit'sja & 0 \\
\hline
\end{tabular}

Es fällt auf, daß es kein unpersönliches 'Passiv' beim analytischen Passiv (Schwedisch, Russisch) gibt - außer im Deutschen. Die Verteilung wird komplementär, wenn wir die Erscheinugn eines ursprünglich inchoativen werden als periphrastische Finitheitsstütze miteinbeziehen. Ein solches werden gibt es nur im Deutschen. Das russische budet, mit dem das deutsche werden oft übersetzt wird, ist ja das Futur der Kopula - und eben nicht äquivalent zu werden. Auf keinen Fall sind die beiden Passivtypen über einen Leisten zu ziehen. 


\subsection{Lexikalische und syntaktische Reflexivierung}

liegen typologisch unterschiedliche Distributionsbedingungen vor: im Schwedischen hat das Reflexivpronomen die thematische Rolle des Objekts zu übernehmen, was in *de träffade sig ausgeschlossen ist. Die syntaktische Reflexivierung dagegen, die offenbar (nach kanonischem Syntaxverständnis) die rückgestufte Agensrolle bindet, ist grammatisch. Das Deutsche hat derartige Unterscheidungen nicht entwickelt: sowohl in der Medialbildung (Die Diva[+TH] unterhält sich[+AG] nicht leicht) als auch bei eigenständiger thematischer Reflexivanapher (Er[+AG] wäscht sich[+TH]) wird das lexikalische Reflexivmorphem verwendet.

Abb. 4: Reflexivmorpheme (z.T. nach Andersson 1998)

\begin{tabular}{||l||l|l||}
\hline & morphologisches Reflexiv & syntaktisches Reflexiv \\
\hline \hline Deutsch & - & sie trafen sich; die Lage verändert sich \\
\hline Niederländisch & - & de situatie verandert $\left({ }^{*}\right.$ zich $)$ \\
\hline Französisch & - & $\begin{array}{l}\text { le sonnet }[+\Theta] \text { s'ecoute de loin; *il }[-\Theta] \\
\text { s'ecoute bien }\end{array}$ \\
\hline Schwedisch & de träffades; läget förändreades & $*_{\text {de träffade sig; *förändreade sig }}$ \\
\hline Russisch & $\begin{array}{l}(\text { oni) vstretilis'; polo ženie izmeni- } \\
\text { los' }\end{array}$ & - \\
\hline
\end{tabular}

Trotzdem bestehen unverwechselbare Übereinkünfte. Die echte, also thematisch unabhängige Reflexivierung im Deutschen ähnelt dem syntaktischen Reflexivpassiv im Schwedischen und Russischen insofern, als eine solche Reflexivierung keine weitere Passivierung mehr erlaubt: aus er wäscht sich kann kein er wird (sich) gewaschen abgeleitet werden. Hier verhindert ein tiefes Koreferenzkriterium bei aller Themarollenverschiedenheit für Subjekt und Objekt (AG $\neq \mathrm{TH})$, daß aus dem aktiven Reflexiv ein Passiv abgeleitet wird. Dies zeigt sich freilich bereits beim Medium, das eine klare passivähnliche Bedeutung und Konfiguration aufweist.

\subsection{Akkusativ des DO beim Passiv}

In (5) und (6) bleibt offen - kann zumindest nicht ausgeschlossen werden -, daß der Objektakkusativ stehen bleibt.

(5) Inget besked-SUBJ/DO gavs oss/vi

Kein(*en) Bescheid wurde gegeben uns/*wir

Der-SUBJ/n-DO Popo wird anständig gewaschen

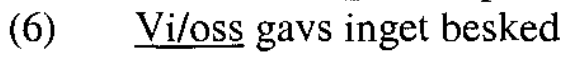

*Wir/uns wurde gegeben kein Bescheid

Abends werden/wird ordentlich die Zähne-SUBJ/DO geputzt 
Dies ist - soferne nicht davon auszugehen ist, daß es sich hier und nur hier um inkorporierte direkte Objekte handelt - unter der 'Passivargumenthypothese' eine sehr kontroverse Erscheinung (frei nach Hegel: diese Erscheinung dürfte es eigentlich nicht geben! (Vgl. Nerbonne 19\%\% in der Auseinandersetzung mit Perlmutters Argumentation auf der Grundlage der Rclationsgrammatik).

\subsection{Besitzverben}

Besitzverben wie haben sind agenslos, jedoch transitiv; im Deutschen gilt streng die Agensvoraussetzung für Passivierung, nicht jedoch im Englischen. Jedenfalls das englische Äquivalent, have, läßt sich passivieren.

(7)a This can be had; this is to be had; this is to have)

- im Unterschied zum Deutschen

b *... kann gehabt werden

c ... ist zu haben

Vgl. aber (7c). Es gibt aber nicht gleich so einsichtig passivische Fügungen wie den Präpositionsinfinitiv zu haben, zu erleiden. Es stellt sich danach die Frage, ob es einen tieferen Zusammenhang zwischen Subjektagens und dem analytischen Passiv bzw. dem dabei verwendeten 2., dem Perfekt-Partizip gibt? Dafür scheint ja die Tatsache zu sprechen, daß die synthetischen passivischen oder passivähnlichen Bildungen dieser agenslosen Transitiva mögich sind.

Abb. 5: Die haben-Passivierung

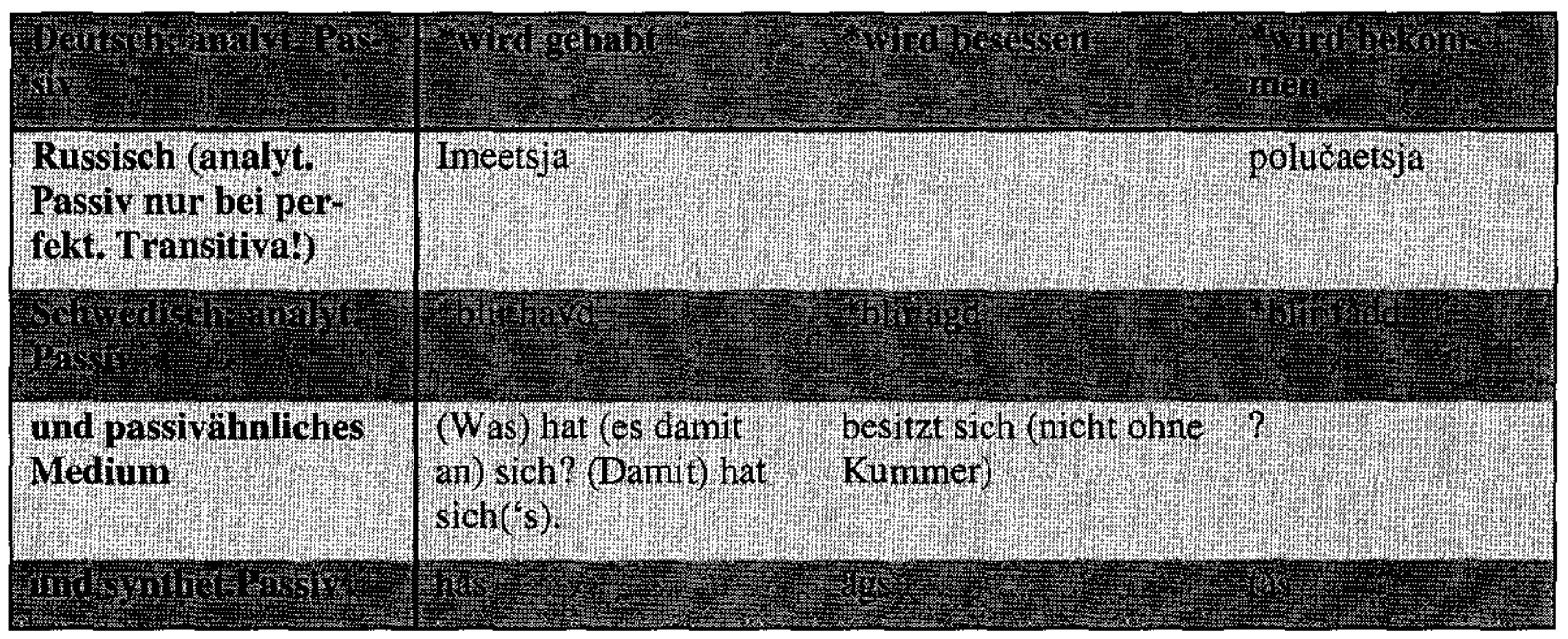

Die Unterschiede zwischen den beiden Passivtypen sind deutlich und unvereinbar. 


\subsubsection{Eingebetteter $(z u+)$ Infinitiv als Schlüssel zum Passivsinn?}

Es stellt sich die Frage, ob das für das Passiv syntaktisch haftbar gemachte Agenschômeur nicht mehr als eine prädikats- und syntaxunabhängige Erklärung gefunden werden muß. Dann nämlich hätte das syntaktisch suspendierte, rückgestufte Agens gar keine syntaktisch erklärende Rolle mehr. Der Weg zu einer solchen Deutung führt über die gesamte PP, nicht über die unter von bzw. durch regierte, rein semantische SOURCE/HERKUNFTS-Rolle.

$$
\text { lassen-Einbettung: }
$$

(a) Ich lasse ihn das machen

(b) Ich lasse das von ihm machen

Aktivinfinitiv

... Passivinfinitiv

Die Aktiv-Passivunterscheidung auf der Grundlage des PPM in (8b) ist jedoch nicht plausibel. Infinitive sind vielmehr wegen der syntaktischen Subjektblockierung (keine Hebung zu einer Tempus- bzw. Kongruenzkategorie im Sinne des Minimalismus) genushybrid - ähnlich wie das PM. Von ihm ist freies Adjunkt mit einer semanytischen HERKUNFTSrolle. Darüber hinaus lassen sich Infinitive nicht ohne weiteres passivieren: vgl. gehört werden/worden sein, jedoch nicht im Imperfekt sowie in den zusammengesetzten Zeiten des Plusquamperfekts und der Vorzukunft. Infinitivpräpositionen scheinen zusätzliche Schranken aufzuwerfen, jedenfalls beim zusammengesetzten Passiv wie im Deutschen - nicht jedoch bei synthetischem Passiv wie im Skandinavischen und Russischen. Das ist erst einmal eine nichttriviale Beobachtung deswegen, weil sie die Argumenthypothese zur Erklärung des Passivs erheblich schwächt.

Abb. 6: Passivsinn ohne Passivmorphologie

\begin{tabular}{|c|c|c|c|}
\hline $\begin{array}{l}\text { nichts ist zu hö- } \\
\text { ren/*gehört zu wer- } \\
\text { den }\end{array}$ & $\begin{array}{l}\text { zu sehen }{ }^{*} \text { gesehen zu } \\
\text { werden }\end{array}$ & zu fühlen $/ * \ldots$ & ist zu merken $/ * \ldots$ \\
\hline $\begin{array}{l}\text { (er is) niets (om) te } \\
\text { horen/*om gehoord } \\
\text { te worden }\end{array}$ & $\begin{array}{l}\text { niets om te zien } / * \text { om } \\
\text { gezien te worden }\end{array}$ & $\ldots$ & $\cdots$ \\
\hline *ist sich zu hören & $* \ldots$ & *ist sich anzufühlen & $* \ldots$ \\
\hline $\begin{array}{l}\text { nothing to hear/be } \\
\text { heard }\end{array}$ & to see/be seen & to feel/be felt & to $($ be) realize $(d)$ \\
\hline inget $*$ blir hört & *blir sett & *blir känt & *blir märkt \\
\hline inget hörs & ses; syns & käns & märks \\
\hline ničego ne slyšitsja & vidneetsja & čustvujetsja & zamečaetsja \\
\hline
\end{tabular}

$\mathrm{Daß}$ dt. *ist sich zu hören ungrammatisch ist einerseits, daß andererseits das Englische die aktive ebenso wie die passive Form setzt und daß das Schwedische und Russische hierfür das Reflexivpassiv verwenden, all dies zeigt, daß eine morphologische Form gesucht wird, die den Passivsinn schon des deutschen nichts ist zu hören und ndl. (om) te horen direkt in die kanonische Passivmorphosyntax umsetzt. Nun tut aber das Deutsche und Ndl. nichts anderes als Suspendierung 
bzw. syntaktische Rückstufung des lexikalisch designierten externen Arguments: entweder durch Infinitheit (keine Hebung zu der Finitheit sichernden funktionalen Kategorie AgrP/TP) oder aber durch Nomenrektion im Infinitiv-PP (vgl. substandardliches zum Hören/Sehen/Anfühlen/ Merken). D.h. zum einen, daß es mehrere, aufs erste gar nicht strukturell vergleichbare Passivsinn erzeugende syntaktische Konfigurationen gibt. Und zum andern evoziert die deutsch/ndl./ engl. Infinitivpräposition $z u /(\mathrm{om})$ te/to lexikalisch eine lokalistische Allativitätssemantik, die. der aspektuellen Inchoativphase bei Perfektiva gleicht und die mit dem Infinitiv in den zu erreichenden Zustand führt. D.h. hier wird wieder Passivsinn über lexikalische Allativität und die Sinnkonfiguration erreicht, die einen Perfektiveffekt erzielt - zweifellos eine nichttriviale Übereinstimmung mit der verfochtenen These, daß Passivität sicher z.T. aus aspektuellen Komponenten ableitbar ist.

\subsection{Vorläufige Zusammenschau}

Die Beispielklassen wurden in Abb. 7 unten so gewählt, daß sich weder lexikalisch noch synthetisch eine semantisch zu verfolgende Handlungsübertragung abbildet.

Abb. 7: Vergleich der Passivierungsformen

\begin{tabular}{|c|c|c|c|c|}
\hline Form & $\begin{array}{l}\text { periphrastisches } \\
\text { Passiv }\end{array}$ & $\begin{array}{l}\text { periphrastisches } \\
\text { Passiv }\end{array}$ & $\begin{array}{l}\text { synthetisches } \\
s \text {-Passiv }\end{array}$ & $\begin{array}{l}\text { synthetisches } \\
\text { sja-Passiv }\end{array}$ \\
\hline $\begin{array}{l}\text { lexikalische } \\
\text { Klassifikation }\end{array}$ & $\begin{array}{l}\text { deutsch } \\
\text { werden }\end{array}$ & $\begin{array}{l}\text { schwedisch } \\
\text { bli }\end{array}$ & Schwedisch & Russisch \\
\hline Besitzverb & - & - & + & + \\
\hline $\begin{array}{l}\text { Verba sentiendi } \\
\text { (agenslos) }\end{array}$ & - & - & + & + \\
\hline $\begin{array}{l}\text { Medium (sich } \\
\text { verändern) }\end{array}$ & - & - & + & + \\
\hline $\begin{array}{l}\text { lexikal. Rezipro- } \\
\text { ka (sich treffen) }\end{array}$ & - & - & + & + \\
\hline $\begin{array}{l}\text { inhaltliche } \mathrm{tV} \\
\text { (beißen, stoßen) }\end{array}$ & - & - & + & + \\
\hline
\end{tabular}

Es ist nicht zu übersehen und aller Wahrschcinlichkeit kein Zufall, daß Passivperiphrastik einerseits und Koreferenz und transitive Handlungsübertragung andererseits in komplementärer Verteilung stehen. Dies gilt es beim folgenden Versuch, das Manipulationsfeld zwischen Aspekt und Genus verbi auszuloten, mitzuberücksichtigen. Haupthürde ist dabei, daß sich die PPM-Bedeutung bei imperfektiven Prädikaten aktivisch, bei perfektiven Prädikaten jedoch passivisch darstellt. Vgl. nochmals (4) oben. In beiden aktionsarttechnisch divergierenden Fällen ist die Grundbedeutung des PPM die eines 'Zustands'. Wie ist Passivsinn aus dieser kategorialen Festlegung ableitbar. Dazu wurde für das Russische der sogenannte 'Perfekteffekt' haftbar gemacht (Schoorlemmer 1995). 


\section{Kategorienbrïcke: 'Perfekteffekt'}

\subsection{Perfekteffekt im Russischen}

Zum Übergangsbereich zwischen Perfektivität und Passivität (man vgl. die prinzipielle Doppeldeutigkeit des PM) gehört die Frage, inwiefern beim PPA im Gegensatz zum PPP so etwas wie die Präsupposition der zum PPM führenden Ereignisverlaufs vorliegt und, sofern dies der Fall ist, wie genau dies beschreibbar ist. Ob wir im Deutschen wie im Russischen davon ausgehen können, daß Aspekt als privatives Oppositionsfeld gesehen werden muß (Maslov 1959; vgl. (3) unten), in dem die tempusstrukturellen Eigenschaften des 'Perfektivs' mit dem des Perfekts übereinstimmen, während der Imperfektiv tempusstrukturell undefiniert bleibt, steht dahin - ist aber angesichts der spezifischen Gültigkeit von (9) auch fürs Deutsche sehr wahrscheinlich.

(9) Perfektivische Prädikate haben immer die Tempusstruktur des Perfekts, also E_R.R,S; dies gilt nicht umgekehrt. D.h. aus E_R.R,S ist keine perfektive Biphasigkeit wie in'(11) bzw. (17a) unten abzuleiten.

Man denke daran, daß aus der Resultatsphase perfektiver Verben immer die dazu führende Inchoativphase auch im tempusreferentiellen Sinne von E_R.R,S impliziert werden kann. Aufgrund der phasisch ungegliederten Ereignisstruktur imperfektiver Prädikate (vgl. (17b)) ist aus der Perfekttempusstruktur E_R.R,S durativer Verben kein Schluß auf Perfektivität zu ziehen. Wir werden gleichwohl sagen, daß auch bei monophasischen (= imperfektiven) Prädikaten im temporalen Perfekt ein aktueller Relevanzbezug, also ein Relevanzbezug auf $S$ (prechaktzeit) vorliegt. Diese Beziehung gilt es auf ihre Verwandtschaft mit (3) oben genauer auszuloten. Dabei ist nicht aus dem Auge zu verlieren. Daß genau der 'oberdeutsche Präteritumschwund' eine Folge gerade dieser tempus-aspekt-strukturellen Verwandtschaft auch bei imperfektiven Verben ist, auf die es hier ankommt (vgl. Abraham 1998). Dazu gehört auch die Tatsache, daß gerade das Perfekt außerordentlich oft Anzeiger von veridikaler Evidentialität ist.

\subsection{Russisches Präteritum ('Imperfekt') perfektiver Verben}

Betrachten wir die Verhältnisse im Russischen aus den folgenden zwei Gründen: Einmal soll der sog. 'Perfekteffekt' sichtbar gemacht werden, der in der Folge eine Rolle spielt, sowie der Zusammenhang zwischen zwischen Aktionsart bzw. aspekt und Tempuskonfiguration; und zum zweiten weiol das Russische gerade mit seiner Aspektverankerung dem Althiochdeutschen sehr ähnelt. Wir erinnern uns an das was wir das 'Passivrätsel' genannt haben: Wie ist die Entwicklung des Verbgenus aus dem reinen Aspektvorgänger vorstellbar. Das heißt aber auch, daß sich jede synchrone Diskussion der Passiverscheinung notwendig der Frage stellen muß, wie die moderne Beschreiung und Erklärung an die alte anschließt. (Trennung von E und R im Sinne von Comrie 1985, Hornstein 1990, Giorgi \& Pianesi 1997)

(10)a Bandity ubili Vanju

Banditen töteten Vanja

b E_R,S = E_SS.R,S

(Schoorlemmer 1995: 240, Beispiel (58))

c E_R_S = E_S.R_S 
wobei $\mathrm{E} \_$_ R die aspektuelle (perfektive) Beziehung und $\mathrm{E} \_\mathrm{S}$ die temporale Vergangenheitsbeziehung im Präteritum zum Ausdruck bringen.

Im Unterschied zum Englischen und Deutschen sind fallen beim Präteritum russischer perfektiver Verben $\mathrm{R}$ und $\mathrm{S}$ nicht zusammen; vielmehr wird das Ereignis von einem Referenzpunkt in der Vergangenheit aus betrachtet, der auf das Ereignis selbst folgt, aber vor S, dem Sprechaktpunkt liegt. Damit ist dieses russische Imperfekt mit der Lesart (4b) gleichzusetzen dem Präsens-Perfekt im Englischen sowie einer der Lesarten des deutschen periphrastischen Perfekts. Die Lesart (4c) dagegen entspricht dem Plusquamperfekt im Englischen und Deutschen. Im Russischen gilt allgemein, daß jedes perfektive Verb gleichgültig in welchem Tempus (ausdrückbar durch eine R:S-Beziehung) stets die Ereignisbeziehung E__S zum Ausdruck bringen.

\subsection{Russisches Präteritum (Imperfekt) imperfektiver Verben}

Wie im Deutschen bei einem Verb jeglicher Aktionsart drückt das Präteritum (Imperfekt)eines imperfektiven russischen Verbs einfache Vergangenheit (Aorist) aus (Schoorlemmer 1997: dort Beispiel (11)).

(11)a E,R_S (?E,_R,S?) = E_S.R_S

$\mathrm{E}$ und $\mathrm{R}$ fallen also zusammen bei imperfektiven Verben und dies wiederum unabhängig vom indizierten Tempus: im Präsens z.B. durch (5b).

(11)b E,R,S = E,S.R,S

Es gelten außer beim perfektiven Verb jedoch auch beim imperfektiven perfektischen Verb Beziehungen zu S, der Sprechaktzeit. Man vgl., was Schoorlemmer zum Russischen beobachtet.

(12)a "The sentence in the perfect, as opposed to the simple past tense, provides a perspective from $\mathrm{S}$, but also the implication that (in very vague terms) the situation in which the event took place still gores on, an implication that is absent in the simple past. I will refer to this implication as perfect effect (as opposed to to perfect temporal structure), abbreviated PE." "[...] precisely those verbs and sentences that deriive PE can derive PPPs (excepting unaccusative verbs) since a PPP obligatorily cooccurs with PE." (Schoorlemmer 1993: 245)

(12)b "Perfective aspect combined with perfective temporal structure optionally triggers PE." (Schoorlemmer 1997: p.251, Beispiel (87)).

(12)c "PE is possible only in a clause showing compositional telicity. [...] Compositional telic ity in Russian is accessible as part of the aspectual system only if no imperfective trigger is present. [...] $\mathrm{PE}$ is derived on the basis of the presence of a specified quantified argument object (internal argument), [+SQA]." (Schoorlemmer 1995: 253, Beispiel (94)).

(12)d "[...] the quantificational properties of the internal argument [...] are crucial for deriving compositional telicity." (Schoorlemmer 1995: 256)

(12)e "Passives always derive PE." (Schoorlemmer 1995: 265)

(12)f "[+PE] is a feature on Aspo." [...] (Schoorlemmer 1995: 272)

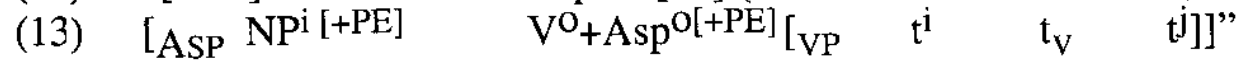


(Schoorlemmer 1995: Beispiel (121)) ... somit Hebung zu einer funktionalen Aspektkate gorie im Einklang mit dem Minimalismus

"Spelling out PE derives a passive." (Schoorlemmer 1995: 277)

\subsection{Perfekteffekt im Deutschen?}

Ich glaube, daß Schoorlemmers Beobachtung zum Perfekteffekt im Englischen und Russischen richtig und wertvoll ist. Aber ich ziehe andere Schlußfolgerungen. Vor allem stelle ich in Abrede, daß einfach direkt aus dem Ausbuchstabieren von PE das Passiv abzuleiten ist (p. 277); vielmehr denke ich daran, daß die Art und Weise, wie der PE je nach Aktionsart des Verbs und nach Hilfsverb und dessen spezifischer Selektionseigenschaft ereignissemantisch realisiert wird, das Verbgenus als Derivat festlegt. Dies gilt es jetzt zu erklären. Der zweite wesentliche Einwand gegen Schoorlemmer besteht darin, daß diese meint, mit ihrem PE jene aspektuellen Eigenschaften bereits identifiziert zu haben, die auch beim Passiv eine Rolle spielen.

Erinnern wir uns nochmals, was unser Forschungshintergrund war: Es geht darum, die aspektuell begründeten Restriktionen beim Genus verbi zu hinterfragen und dabei besonders der Frage nachzugehen, ob - in Teilen der Passivgrammatik des Deutschen oder vielleicht sogar insgesamt - das Passiv eine von Aspekteigenschaften abgeleitete Konstruktion ist.

\subsection{Aspektphasen und Aktionsartprädikation}

Ich schließe formal-begrifflich, nicht jedoch inhaltlich an Abschnitt 8 oben an. Die für unsere Ziele entscheidende Aktionsartopposition zwischen terminativen (perfektiven) und interminativen (imperfektiven) Prädikaten kann wie folgt beschrieben werden (so Abraham 1989 und verschiedentlich später).

bi-pasische Terminativa: versenken $\left[\mathrm{AG}_{\mathrm{i}}, \mathrm{TH}_{\mathrm{j}}\right]$ versenkt $\left[\mathrm{TH}_{\mathrm{j}}\right]$<smiles>[3H][V]C=[V]</smiles>

b monophasische Interminativa:
Tempusstruktur für PPM:

E_R,S bzw. E_R.R,S

E,R_S bzw. R_S. E,R

Die in Abschnitt 8 oben definierten Begriffe beziehen sich auf t" in (15a). Für Imperfektiva gibt es keinen Kulminationspunkt bzw. Folgezustand in Parsons' Sinne von (12)-(14) unten. Ich gehe weiter davon aus, daß jede Periphrastik im Deutschen sowohl semantisch wie auch syntaktisch kompositional $\mathrm{zu}$ beschreiben ist und daß Erklärungen, was grammatische und was ungrammatische Bildungen sind, in dieser Kompositionsmechanik ruhen. Kompositionen sind Ergebnisse von Projektionen einer Tempus- bzw. Aspektstruktur auf eine andere. Dabei geht von der jeweils skopusübergeordneten Kategorie Projektionspriorität aus. Dieser Mechanismus entspricht sowohl den deutschen Linearitätsverhältnissen der funktionalen Verbkategorien (Kongruenz-, Tempus, Modus- und Genuselemente), und sie spiegelt sich einzelsprachlich durchaus verschieden in der Abfolge der funktionalen syntaktischen Knoten. (16) zeigt, wie dies zu denken ist.

$$
[\mathrm{VP} \ldots[\mathrm{v} \text {-FIN }[\mathrm{v} \text {-FIN wird }][\mathrm{PPM} \text { versenkt]]] }
$$




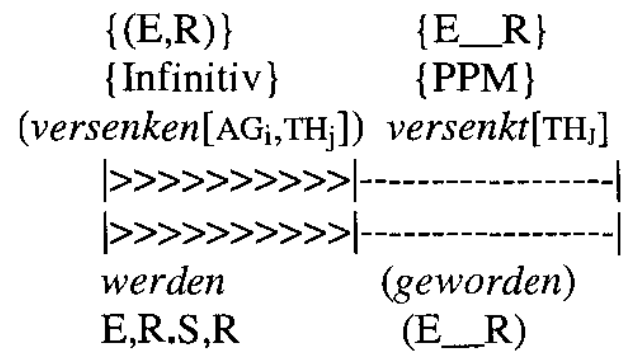

$\{(\mathrm{E}, \mathrm{R})\}$

$\left\{\mathrm{E} \_\mathrm{R}\right\}$

senken $\left.\left[\mathrm{AG}_{\mathrm{i}}, \mathrm{TH}_{\mathrm{j}}\right]\right)$ versenkt $\left[\mathrm{TH}_{\mathrm{J}}\right]$

$|>>>>>>>>--------------|$

E,R.S,R (E_R)
Tempusstruktur:

E,R $\quad$ E__R

Aktionsartachse:

(versenken) $\leftrightarrow \quad$ versenkt $[+$ perf $]$

werden $\leftrightarrow$ (geworden)

C-"anchored" (Enç 1987, Hornstein 1990)

$\therefore$ wird gerade versenkt $\wedge$ wird versenkt sein

(Implikation alleine aufgrund der perfek

tiven AA)

Aus (18) folgt, daß die finite Komponente mit (E,R.)S,R mit sein oder werden mit S-Projektion (Sprechaktzeit) auf E,R bzw. E__R situiert ("S-anchored") ist, d.h. hier mit wird auf die 1. Phase des Vollverbs projiziert. Während das Hilfsverb S auf der AA-Achse verankert, bestimmt V mit $\{$ E,R.E_R $\}$, welche Implikationen gültig sind.

Wie sieht die Projektion bei Imperfektiven aus. Vgl. wir (17). In (16) wie in (17) trenne ich zwischen Tempusstruktur in der Reichenbachnotation und AA-Konfiguration.

[vp $\ldots[$ [v-FIN $[\mathrm{v}$-FIN wird] [PPM unterstützt]]]

$\{(\mathrm{E}, \mathrm{R})\} \quad\left\{\mathrm{E} \_\mathrm{R}\right\}$

\{Infinitiv $\} \quad\{\mathrm{PPM}\}$

(unterstützen $\left[\mathrm{AG}_{\mathrm{i}}, \mathrm{TH}_{\mathrm{j}}\right]$ ) unterstützt $\left[\mathrm{TH}_{\mathrm{J}}\right]$

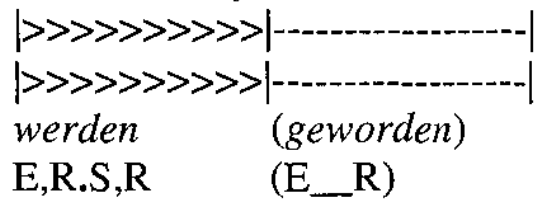

Tempusstruktur:

E,R $\quad$ E__R

Aktionsartachse:

(unterstützen) $\leftarrow \quad$ unterstützt[-perf]

werden $\leftrightarrow$ (geworden)

$\therefore$ wird gerade versenkt (ohne Implikation eines erreichten Zustands: imperfektive AA bei unterstïtzen)

Dies zeigt aber, das dasjenige, was Schoorlemmer (1995: 245 et passim) 'Perfekteffekt' genannt hat (und was so etwas wie den Gegenwartsbezug des Present perfect nach einem zurückliegenden Ereignis bedeutet), mit (16)-(17) gar nicht genau referiert ist. Hier handelt es sich um etwas ähnliches und doch anderes - nämlich grob gesagt den Umstand, daß sowohl aus dem Perfektpartizip (PPM) perfektiver Verben wie auch imperfektiver Verben der zu dem Partizipzustand führende Vorvorgang erschlossen wird. Wählen wir dazu den Terminus 'Perfektiveffekt'. Der volle Umfang dieses Begriffs läßt sich in dem Unterschied der beiden AA-Konfigurationen und den Tempusstrukturen darstellen. In (16) bzw. (17) zeigt sich der hier angesprochene 'Perfektiveffekt' als echte Implikation des PPM bei [+perf]-Prädikaten, also allein aufgrund der AA-Struktur als 'Perfekteffekt'. Bei [-perf]-Verben dagegen ist für das PPM keine derartige Implikation mehr gegeben (man vergleiche die fettgedruckten Implikationspfeile in der AA-Konfiguration in (16)(17)). Ich gehe jedoch davon aus, daß jedes PPM unabhängig von seiner lexikalisch inhärenten AA-Struktur dieselbe Tempuskonfiguration hat: nämlich ebenfalls E_R. Dies sei, so möchte ich annehmen, die schwächere Version des AA-begründeten Perfektiveffekts. Sie zeigt sich darin, daß man nicht im russischen Sinne (im reinen Sinne eines 'Präsensperfekts') von einem 
Anhalten des Resultatszustands des PPM bis zu S herauf sprechen kann; vielmehr besitzt der PPM-Zustand 'Relevanz' für S. Dies ist eher eine Eigenschaft der Tempusstruktur als der AAKonfiguration: vgl. E,R.R__S für das reine Präteritum sowie die Entsprechungen des wohl entperfektivierten analytischen Präteritums beim 'oberdeutschen Präteritumschwund' - gegen E__R.R,S beim englischen Präsensperfekt - mit der Bedeutung eines Relevanzperfekts, wenngleich der genaue Unterschied zwischen Präsensperfekt und Relevanzperfekt in der Aktionsartkonfiguration und der Tempusstruktur nach Reichenbach nicht zum Ausdruck kommt.

\subsection{Begriff des 'erweiterten Jetzt' sowie des 'Folgezustands'}

Der Perfekteffekt Schoorlemmers (1995) läßt sich ebenso wie der hier referierte und erklärte Perfektiveffekt des PPM auf der folgenden Grundlage nicht nur z.T. formal fassen, sondern auch und gerade dies ist mir in diesem Zusammenhang wichtig - mit aspektueller Perfektivität in deutlichen Bezug setzen. Vgl. (11) und (17). Ich berufe mich bei der Diskussion der folgenden Tempusbegrifflichkeit auf Arbeiten wie Dowty (1979), Parsons (1990) sowie Giorgi \& Pianesi (1997). Dowtys 'extended-now theory' ist in der Begrifflichkeit der Intervallsemantik beschrie ben. Genauer gesagt ist das 'ausgedehnte Jetzt' ein Zeitprädikat $X N(t)$, das gilt zu $t$ ' dann und nur dann, wenn zwischen $t$ und $t$ ' eine bestimmte temporale Beziehung besteht (G\&P 90).

Definition ((51) bei G\&P 1997) " $X N(t)$ gilt zum Zeitpunkt $t$ ', wwenn $t$ ' ein Schlußsubintervall des durch $t$ bezeichneten Intervalls ist."

Bei Parsons ist ein ähnlicher Begriff entwickelt worden: der des Folgezustands (consequent, or resultant, state; abgekürzt FZ). Ein Satz wie (13a) hat die logische Form wie in (13b),

(19)a Johann hat einen Apfel gegessen

b $\exists e \exists x($ ess- $(e) \wedge \operatorname{Agens}(e, \operatorname{Johann}) \wedge \operatorname{Patiens} / T h e m a(e, x) \wedge \operatorname{Apfel}(x) \wedge \operatorname{gilt}(F Z(e), S))$

wo FZ (für Folgezustand) eine Teilfunktion von Ereignissen auf Ereignisse ist, die jedem Ereignis $e$ seinen Folgezustand zuordnet ('gilt' als solches Zuordnungsprädikat). (13) heißt also u.a., daß zum Sprechaktzeitpunkt, $S$, ein Folgezustand für das Ereignis gilt. Solche Folgezustände sind ausschließlich für Ereignishöhe- oder -endpunkte definiert. Es gilt dann weiter (vgl. G\&P 92; 93; 98):

(20)a Der Folgezustand (FZ) zu $t$ gilt, wwenn $e$ zum Zeitpunkt $t$ oder irgendwann vorher seinen Höhepunkt ( $\mathrm{Culm})$ erreicht.

b $\forall e \forall t(C u l m(e)$ v $e \leq t \rightarrow \operatorname{gilt}(\mathrm{FZ}(e), t))$

c $x$ ist a Folgezustand (FZ) von $e$ dort, wo $e$ topologisch geschlossen ist $=_{\mathrm{df}}$ Linksgrenze $(x)$ $=$ Rechtsgrenze $(e)$; vgl. (11), (17a) zum Unterschied von (17b)

Der semantische Beitrag eines PPM besteht in einer Beziehung $F Z\left(e^{\prime}, e\right)$ zwischen zwei Ereignissen $e^{\prime}$ und $e$, wwenn $e^{\prime}$ der Folgezustand von $e$ ist. Hilfsverben wie haben, werden, sein nehmen Ereignisvariablen und, soferne sich PPM-Sätze mit Hilfsverben verbinden, gehen die Ereignisvariablen in eine derartige Beziehung mit ihnen ein, daß $e^{\prime}$ Schlußteil, $\rho$, von $e$ ist: somit $\rho\left(e_{A u x}, e^{\prime}\right)$. Der Beitrag der Partizipialmorphologien besteht darin, das durch VP denotierte Ereig- 
nis zu einem Ereignishöhepunkt und einem topologischen Schluß zu führen und einen zweiten Ereignisteil einzuführen, nämlich den Folgezustand.

(21)a Montag hatte Johann das Rennen gewonnen

b (Bis heute) hat Johann das Rennen noch nicht gewonnen

(22)a $\exists e^{\prime} \exists e$ (gewinn $(e) \wedge \operatorname{Agent}(e, x) \wedge \mathrm{CS}\left(e^{\prime}, e\right) \wedge \rho\left(\right.$ Montag, $\left.\left.e^{\prime}\right) \wedge \operatorname{Montag}<S\right)$

b $\neg \exists e^{\prime} \exists e\left(\right.$ gewinn $\left.(e) \wedge \operatorname{Agent}(e, x) \wedge \operatorname{CS}\left(e^{\prime}, e\right) \wedge \rho\left(S, e^{\prime}\right)\right)$

Im Falle von (21a) gibt es ein Gewinn-Ereignis und einen Folgezustand $e^{\prime}$ derart, daß $e^{\prime}$ in der $\rho$ Relation zu Donnerstag steht. In (21b) dagegen gibt es für keinen relevanten Ereignisteil einen Folgezustand - d.h. in der $\rho$-Relation mit dem Sprechaktzeitpunkt $S$.

Ich definiere hier nur sehr grob die angesprochene 'Vorgängerphase' zum PPM $(=F Z(e)$, $t$ ). Dies erfolgt in naher Anlehnung an (20a-c). Vgl. (20d-g).

(20)d Eine Vorgängerphase (VOR) gilt zu $t$, wwenn $e$ zum Zeitpunkt $t$ oder irgendwann vorher seinen Höhepunkt erreicht.

e $\forall e \forall t(C u l m(e) \vee e \leq t \rightarrow$ es gilt $(F Z(e), t))$

f Redundanzregel: $F Z(e, t) \rightarrow \operatorname{VOR}(e, t-n)$

$\mathrm{g} x$ ist eine Vorgängerphase, $\operatorname{VOR}(e, t-n)$ von $F Z(e, t)$ dort, wo $e$ topologisch geschlossen ist $=_{\mathrm{df}}(\operatorname{Rechtsgrenze}(e)=$ Linksgrenze $(x))$.

(20d-g) scheidet Eigenschaften (also Zustände ohne Vorgängerphase) von 'erreichten Zuständen'; es läßt allerdings offen, ob die Vorgängerphase lexikalisch oder grammatisch impliziert ist. $(20 \mathrm{~g})$ im besonderen dreht die Relation in (20c) um, da die Perspektive auf das Gesamtereignis $e$ endend mit dem Zustand ausgedrückt durch das PPM von der Vorgängerphase (VOR) aus entwickelt wird.

\section{7. 'Unakkusativität' und seine aspektuelle Ableitung}

Ergative oder unakkusativische Verben haben als eigene Verbkategorie nur unter der Argumenthypothese Bestand. Unter der Aspekthypothese sind sie bloß Intransitiva einer bestimmten Gattung. Ich habe unter Berücksichtigung einer umfassenden Datenzusammenschau dafür plädiert, eV mit perfektiven Intransitiva gleichzusetzen (Abraham 1993, 1995, 1998(1999)). Wie nun sind iperfV $(=$ ' $\mathrm{eV}$ ') tempus- und aktionsartkonfigurational darzustellen? Gehen wir dabei von dem zentralen Datenbefund aus, daß Unakkusativa nicht passiviert werden können. Was sich bei Passivrückstufung ergeben müßte, wäre eine unpersönliche Struktur, eben ein unpersönliches 'Passiv'. Vor dem hier vertretenen Aspekthorizont müßte also der Umstand, daß sich zu Unakkusativa kein unpers. 'Passiv' bilden läßt, als Sekundärerscheinung aus der speziellen Ereigniskonfiguration ableitbar werden.

Blicken wir zur Ereignissemantik. Wir haben bisher gesagt, daß jedes Passiv aus der Projektion finiter Hilfsverben auf Sekundärphase des Gesamtereignisses (Resultats- bzw. PPMPhase) besteht. Jedes PPM - und insbesondere ein perfektives - ähnelt somit semantisch einem ' $\mathrm{eV}$ '. ' $\mathrm{eV}$ ' drücken einen kurzen bis auf einen Punkt reduzierten inchoativen Vorgang unter Einschluß der Resultatsphase aus. Diese beiden Ereigniseigenschaften vereinigen sich nur im Übergangspunkt zwischen der Ereignisvorphase und der PPM-Phase. Dabei sind allerdings in jedem Fall zweierlei perfektive Intransitiva zu unterscheiden: 
(23) iperfV mit ausgedehnter Inchoativphase: das sind solche mit Zielobjekt bzw. telischer Adverbphrase, also etwa das Haus bauen; durch den Flu $\beta$ schwimmen; über den graben springen; untergehen. Der Infinitiv dieser Prädikate denotiert den Vorgang der Inchoativphase UND den Übergangspunkt zum implizierten Resultat.

(24) iperfV, bei denen die Inchoativphase mit der Resultatsphase zusammenfällt: dies geschieht nur im Übergangspunkt, der Eigenschaften beider Phasen besitzt. Dies seien u.a. die punktuellen iperfV entschlafen, ankommen, sterben, versinken, finden.

Die Aktionsartprojektion des inchoativen Hilfsverbs werden projiziert sich in diesen Übergangspunkt - was wegen der Ausdehnungslosigkeit des Übergangspunktes zu keiner sinnfälligen Lösung führt.

Wie stellt sich der Unterschied zwischen (1) und (2) ereignisstrukturell dar? Prüfen wir zuerst, wo sich Passivsinn ergibt - ein solcher Unterschied wäre dann ereignisaspektuell zu erklären.

(25) Es wurde in den Graben gesprungen, sein Haus gebaut, durch den Fluß geschwommen

(26) *Es wurde gefunden, angekommen, versunken, verlöscht, eingetreten, ausgelöst, gestorben (gleichgültig ob mit oder ohne (Inhalts-)DO (demnach auch bei unpersönlichen Passiven von $\mathrm{tV})$.

(27) ad (3):

sterben/finden/ankommen

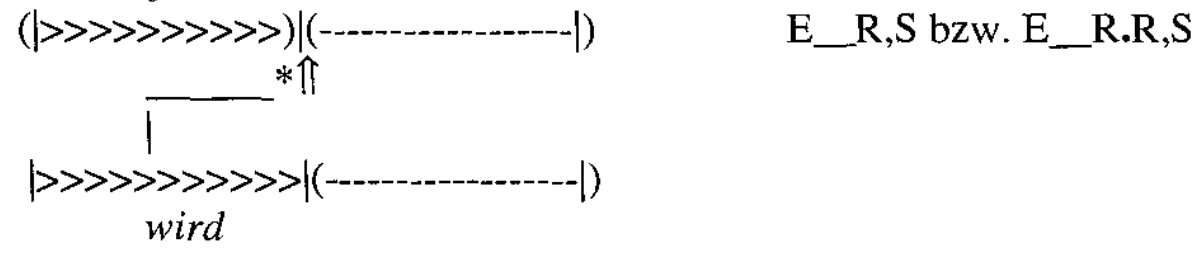

(28) ad (4) springen in

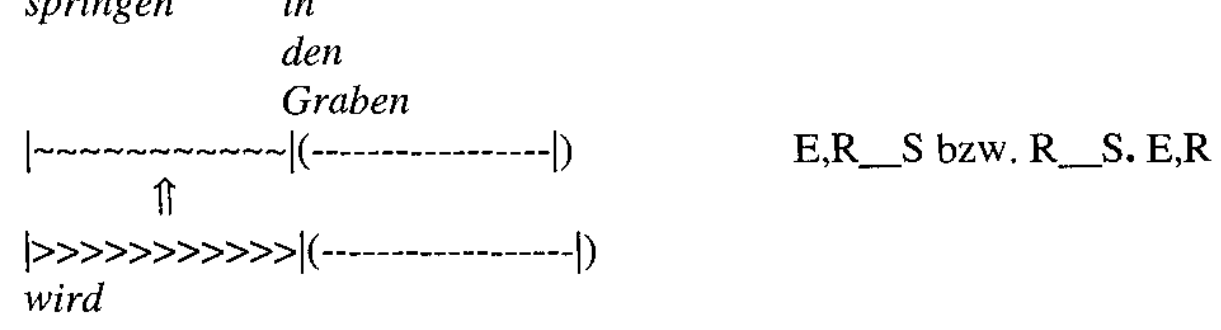

Der Projektionspfeil in der Ereignisgraphik, , spiegelt sich in der syntaktischen Ableitung als Inkorporationsanhebung. Vgl. weiter unten. Was hier zu beweisen war: Die Passivunfähigkeit von ' $\mathrm{VV}$ ' kann auf der Grundlage der Aspekthypothese erklärt werden.

Eine bedeutsame Rolle in Schoorlemmers Argumentation spielt der grundlegende, nur diathetisch zu erklärende Unterschied zwischen unakkusativischen und unergativischen Prädikaten (1993 Kap. II,2.2). Unakkusative seien anders als Unergative insofern, als sie in aspektuell identischen Transitivsätzen vorkommen (263). $\mathrm{Zu}$ den diagnostischen Prüfwerten zählt sie - im Einklang mit dem Marktkanon - das unpersönliche Passiv, das nur von Unergativen möglich sei. Ich bestreite diese Feststellung in seiner Empirie - und will gleichzeitig zeigen, daß die Be- 
schränkungen tatsächlich aspektuell erklärbar sind ('(partielle) Aspekthypothese zur Passivierung'). Es sei dabei erinnerlich, daß Passivierungen (mit 2. Partizip) sich im Deutschen (und Ndl. und Fries.) auf Prädikate mit einem externen Argument beschränken, im Englischen und Russischen auf solche mit externem und internem Argument ('Argumenthypothese zur Passivierung').

Orientieren wir uns an zwei Schlhsselbeispielen und Grammatizitätsurteilen zum 'unpersönlichen' Passiv.

'ERGATIVISCHE/UNAKKUSATIVISCHE' (/ TERMINATIVE, PERFEKTIVE INTRANSITIVE) PRÄDIKATE:

(29)a 'Es wird (jetzt gerade[E,R.S,R]/schon noch einmal[E_R.R,S]) angekommen (werden)

b ${ }^{* *}$ Es ist angekommen

c Es wurde im 1. Weltkrieg mit Inbrunst gestorben

d ${ }^{* *}$ Es war mit Inbrunst gestorben

e ${ }^{* *}$ Er wird angekommen

f Er ist angekommen (*worden)

'UNERGATIVISCHE' (/ IMPERFEKTIVE INTRANSITIVE) PRÄDIKATE:

(30)a Es wird gegangen (werden)

b Es ist gegangen

c ${ }^{(*)}$ Er wird gegangen

Auffällig ist nun: einmal daß es gar nicht so unmöglich zu sein scheint, daß selbst 'Ergative/Unakkusative' unpersönliche Passive bilden; und zum zweiten daß (10c) in einer bestimmten, nämlich terminativen Lesart möglich sind, wogegen die Entsprechungen bci ankommen;sterben keine Lesartmöglichkeiten bietet.

Ich erkläre die Grammatizitätserscheinungen auf der Grundlage der kompositionellen Ereignisphasensemantik. Es ist richtig, daß perfektive Intransitiva wie ankommen, sterben nicht ganz plausibel passiviert werden - aber es ist eben nicht ausgeschlossen. Warum das eine, warum das andere? Wird denotiert stets den Inchoativvorgang, der zum Resultatsstand fhhrt. Wird hat kompositionelle Projektionspriorität hber der Projektion des Partizips angekommen. D.h. wird sucht sich bei der semantischen Komposition in der Ereignissemantik des eingebetteten angekommen die seiner Ereignissemantik entsprechende Inchoativphase. Diese ist von angekommen jedoch bloß impliziert, nicht direkt denotiert (Klammer um Inchoativphase).

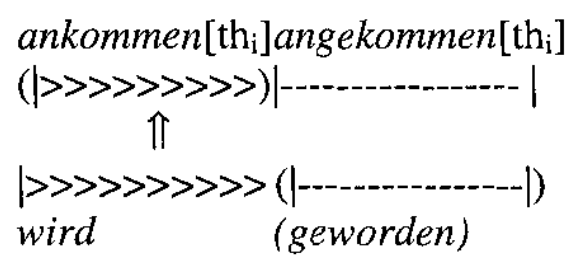

Es ist vielleicht zu betonen, daß das rückgestufte Agensargument dabei die 'transitive' thematische Rolle spielt, die die erforderliche Phasensättigung (Argument zu beiden Ereignisphasenprädikaten) erfhllt. (10b) ist, wenn man sich auf die Lesartkontexte für (9) eingestellt hat, ebenso möglich. Im letzten Falle ist der Perfektiveffekt besonders deutlich: die Resultatsphase impliziert die Inchoativphase. Zu beachten ist, daß nach dem 'Aspektstandpunkt' die sog. 'Ergative' bzw. 'Unakkusative' nur deswegen ein objektartige Subjekte haben, weil sie als perfektive Intransitiva 
beide Ereignisphase mit einem einzigen Argument absättigen; da ein (Resultats-)Zustand nie ein Agens, sondern stets bloß ein Patiens/Thema als Argument erlaubt, ist kein Passiv möglich. Dies hält auch unter der 'Argumentpassivhypothese' stand.

Aber weshalb ist nun (9f völlig ungrammatisch? Man beachte: Nach der 'Argumenthypothese' wird die Ungrammatikalität von (9f) auf identische Weise erklärt: nämlich allemal, d.h. ohne Grammatikalitätsunterschiede zulassend, durch die Unakkusativitätshypothese, d.h. "ein einmal vom internen zum externen vorgestuftes Argument ist syntaktisch immobil geworden'. Unakkusativität ist somit wie eine lexikalische Passivdiathese. Man kennt typologisch keine syntaktische Diathese, die durch mehr als eine aneinandergereihte Valenzreduktion entstehen kann. Das mag so sein - ist immerhin empirisch offen und eben durch keinerlei andere Logik 'unhintergehbar' gemacht. Es mag also auch nicht so sein müssen. Unter der 'Aspekthypothese' jedoch lassen sich solche Grammatikalitätsunterschiede anders - besser? - erklären. Der Grund fhr die gravierende Falschheit von (9f) etwa mag daran liegen, daß perfektive Verben bereits im synthetischen Präsens - also bei [E,R.S,R] - die erste, die Inchoativphase denotieren. Durch werden entsteht abermals eine Projektion auf die Inchoativphase, sozusagen gedoppelt. Das mag der ereignissemantische Grund fhr die Ungrammatikalität sein. Wir haben, so scheint es, noch nicht hinreichend empirisch-typologischen Überblick in der ereignissemantischen Beschreibung, um ermessen zu können, ob eine so beschriebene Doppelprojektion systematischen Erklärstatus besitzt.

Wichtig bei (10) ist die Tatsache, daß bei der metaphorischen (und idiomatisch überaus populären) Lesart die perfektive Verbpartikel hinaus-/raus- dazugedacht wird - wobei zugegebenermaßen unklar bleibt, ob die elliptische, präsupponierte Agensrolle der Passivierungsauslöser ist oder die elliptische terminative Verbpartikel hinaus-/raus-entscheidend ist.

\section{Daten zur Entscheidung}

\subsection{Verteilungsbedingungen im Russischen und im Deutschen}

Das Russische ist ein ausgezeichneter Prüfstein für die hier zum Denotat des PPM entwickelten Erklärbereichs, dies aus mehreren Gründen: zum einen weil Perfektiv- und Imperfektivprädikate morphologisch geschieden erscheinen und sich die implikativen folgerungen von den implikaturellen gut trennen lassen; zum zweiten weil sich die Bildung des PPM überhaupt an Aspekt bindet; und zum dritten zeigt sich eine Interaktion zwischen Tempus und Aspekt, die für die in diesem Aufsatz angesprochenen Fragen augenöffnend wirken

zu Abb. 8: 'Russisch - die Präsenslücke in einer Aspektsprache' vgl. die Folgeseite 
Abb. 8: Russisch - die Präsenslücke in einer Aspektsprache

\begin{tabular}{|c|c|c|c|c|}
\hline & & Prateritum & Prasens & Futur \\
\hline erfekti & $\begin{array}{l}\text { Aktiv } \\
\text { (Reflexiv) Passiv: } \\
\text { keine periphrasti- } \\
\text { sehen Fommen }\end{array}$ & $\begin{array}{l}\text { otkryval } \\
\text { offnete } \\
\text { ot kryvalsja } \\
\text { offnete sich? }\end{array}$ & $\begin{array}{l}\text { otkryvaet } \\
\text { offnet } \\
\text { otkryvaetsja } \\
\text { offinet sich? }\end{array}$ & $\begin{array}{l}\text { budet otkrywat } \\
\text { wird offnen } \\
\text { buidet otkpy val sia } \\
\text { wird sich offnen }\end{array}$ \\
\hline & Aknv W & orkryi & $\begin{array}{l}\text { 0. durch } \\
\text { E R keine } \\
\text { S Veranke- } \\
\text { fung moglich }\end{array}$ & $\begin{array}{l}\text { otkroet } \\
\text { wird of nen }\end{array}$ \\
\hline & Passiv: & by wurde geof net & 0 & $\begin{array}{l}\text { budet otkryt } \\
\text { swird geoff } \\
\text { sein }\end{array}$ \\
\hline & Reflexiy & $\begin{array}{l}\text { otkryls ja } \\
\text { offnete sich" }\end{array}$ & & $\begin{array}{l}\text { otkroetsja } \\
\text { ofra sich offnen }\end{array}$ \\
\hline & Perfekt & otkryt worden $(i s t)$ geoff & & 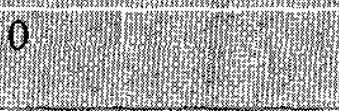 \\
\hline & $\begin{array}{l}\text { Perfektiv-Passiv, } \\
\text { jedoch mit Präsens } \\
\text { vertretung }\end{array}$ & $\begin{array}{l}\text { byl oukyt wurde } \\
\text { gecoffnet }\end{array}$ & $\begin{array}{l}\text { otkyt } \\
\text { geoffinet nur } \\
\text { bet Transi- }\end{array}$ & $\begin{array}{l}\text { budet otkryt wire } \\
\text { geoffnet sein? }\end{array}$ \\
\hline
\end{tabular}

Diese Oppositionsfelder des Russischen unterscheiden sich grundlegend vom Deutschen - das ganze Bild hat jedoch insgesamt doch Gemeinsamkeiten. Vgl. Abb. 9.

Abb. 9: Deutsch - persönl. Diathesen und unpersönl. 'Passivdiathese' (eA=externes Argument; $\mathrm{e}_{1}=$ lex. Designiert, $\mathrm{e}_{2}=$ syntaktisch abgeleitet ('Passivsubjekt')

\begin{tabular}{|c|c|c|c|c|c|c|}
\hline & \multicolumn{3}{|c|}{ Vorgang } & \multirow{2}{*}{$\frac{\text { Zustand }}{\text { Perfekt }}$} \\
\hline & & & Präteritum & Präsens & Perfekt & \\
\hline \multirow[t]{4}{*}{$\begin{array}{l}\text { Imper- } \\
\text { fektiv }\end{array}$} & \multirow[t]{2}{*}{ Aktiv } & $\mathrm{tV}$ & $\begin{array}{l}+ \\
\text { schob }\end{array}$ & $\begin{array}{l}+ \\
\text { schiebt }\end{array}$ & $\begin{array}{l}+ \\
\text { hat geschoben }\end{array}$ & *ist geschoben \\
\hline & & iV & $\begin{array}{l}+ \\
\text { lief }\end{array}$ & $\begin{array}{l}+ \\
\text { läuft }\end{array}$ & $\begin{array}{l}+ \\
\text { ist(/hat) gelaufen }\end{array}$ & *ist gelaufen \\
\hline & \multirow[t]{2}{*}{ Passiv } & $\mathrm{tV}$ & $\begin{array}{l}+ \\
\text { wurde ge- } \\
\text { schoben }\end{array}$ & $\begin{array}{l}+ \\
\text { wird } \\
\text { geschoben }\end{array}$ & $\begin{array}{l}+ \\
\text { ist geschoben } \\
\text { worden }\end{array}$ & *ist geschoben \\
\hline & & iV & $\begin{array}{l}+ \\
\text { es(EXPL) } \\
\text { wurde ge- } \\
\text { laufen }\end{array}$ & $\begin{array}{l}+ \\
\text { es(EXPL) } \\
\text { wird ge- } \\
\text { laufen }\end{array}$ & $\begin{array}{l}+ \\
\text { es(EXPL) ist ge- } \\
\text { laufen worden }\end{array}$ & $\begin{array}{l}\left.\text { (es ist gelaufen }{ }^{1}\right) \\
\text { *es ist gegangen }\end{array}$ \\
\hline
\end{tabular}




\begin{tabular}{|c|c|c|c|c|c|c|}
\hline \multirow[t]{4}{*}{ Perfektiv } & \multirow[t]{2}{*}{ Aktiv } & $\mathrm{tV}$ & $\begin{array}{l}+ \\
\text { zog heraus }\end{array}$ & + & + & + \\
\hline & & iV & $\begin{array}{l}+ \\
\text { kam an }\end{array}$ & + & + & + \\
\hline & \multirow{2}{*}{$\begin{array}{l}\text { Passiv: } \\
\mathrm{e}_{2}= \\
\text { Passiv- } \\
\text { subjekt } \\
\text { ohne } \\
\text { Subjekt }\end{array}$} & tV & $\begin{array}{l}+ \\
\text { wurde her- } \\
\text { ausgezogen }\end{array}$ & - & + & + \\
\hline & & $\mathrm{tV}$ & $\begin{array}{l}\text { es wurde } \\
\text { herausgezo- } \\
\text { gen }\end{array}$ & $\begin{array}{l}\text { es wird } \\
\text { herausge- } \\
\text { zogen }\end{array}$ & + & - \\
\hline & $\begin{array}{l}\text { ohne } \\
\text { Subjekt }\end{array}$ & iV & $\begin{array}{l}\text { *es(EXPL) } \\
\text { wurde ange- } \\
\text { kommen }\end{array}$ & - & - & $\begin{array}{l}\text { *es(EXPL) ist angekom- } \\
\text { men }\end{array}$ \\
\hline
\end{tabular}

${ }^{1}$ : es ist gelaufen gehört zur Struktur, wo gilt $\mathrm{e}_{1}=\mathrm{e}_{2}$ - was ja auf jeden Fall ausgeschlossen ist und nur rein lexikalisiert zu verstehen ist.

Die auffälligen Distributionsbeschränkungen im Deutschen sind die folgenden:

Abb. 10: Verteilung zwischen Vorgangspassiv (mit werden) und Zustands'passiv' (mit sein)

\begin{tabular}{|l|l|l|}
\hline & IMPERFEKTIV & PERFEKTIV \\
\hline Zustandspassiv & $*_{\text {ist geschoben }}$ & X ist herausgezogen \\
\hline Vorgangspassiv & $* e s(\mathrm{EXPL})$ wird angekommen & $*$ es(EXPL) wird gelaufen \\
\hline
\end{tabular}

wobei das ZuP perfektiven Verben vorbehalten ist; vgl. (32).

\section{PM}

$(|>>>>>>>>>|)-----\cdot-------\mid$

$<$ werden $>$ (geworden)

$$
<\text { sein }>
$$

und wobei weiter:

(33) a $P P M+$ sein nicht jedoch:

b $P P M+$ worden sein $\quad \Rightarrow P P M+$ sein

Es gelten die folgenden Selektionsregeln:

$$
\begin{aligned}
& \Rightarrow P P M+\text { worden sein bei }[+ \text { perf }] \text { sowie } \mathrm{E} \_\mathrm{R} \\
& \text { bei [-perf], es sei denn über }
\end{aligned}
$$


(34) DEUTSCH:

(a) PPM[-passiv]+haben: $\quad \mathrm{tV}$

(b) PPM[+passiv]+sein: $\quad$ iV[+perfektiv]

(c) PPM[+passiv]+werden: $\quad$ iV $[ \pm$ perfektiv $]$

(d) PPM[-passiv]+sein: $\quad$ FBV-iV wie laufen, gehen, schwimmen, vor allem wenn telisch konnotiert

(e) PPM[-passiv]+haben: $\quad$ iV[-perfektiv] wie schlafen, träumen

NIEDERLÄNDISCH:

(f) PPM[-passiv]+zijn d.h. neben de kar werd geschoven auch is geschoven; dazu gehört, daß das PPM von worden in aller Regel fehlt: $I k$ ben

er verslagen (geraakt/*geworden).

(g) $\mathrm{tV}[+$ perf]: beginnen, verliezen, vergeten - alle (+DO!): ik ben mijn pm. vergeten/verloren

Ndl. (f) beweist direkt, daß der Perfekteffekt eine Stufe in der Grammatikalisierung der Passiverscheinung ist: Was im Deutschen Zustandspassiv ist und Aspektbeschränkungen unterliegt, hat sich im Ndl. als Möglichkeit durchgesetzt, das Vorgangspassiv wiederzugeben und zwar ausschließlich auf der Grundlage des erschlossenen Vorgangs zum weiter aspektuell nicht eingeschränkten PM.

Was aus diesem stark aspektuell begründeten PPM-Identifikationsszenario herausfällt, ist werden+PPM - das ja von Aspektunterscheidungen völlig absieht! Wir haben die Position verfochten, daß dies in einem nicht aspektbegründeten Szenario auch über die Tempusstruktur einzubringen ist: daß also auch bei imperfektiven PM gilt E__R. Dies ist der Perfekteffekt: es wird die Vorphase zum PM mit Relevanzeffekt für R,S bzw. E,R.R,S impliziert.

\subsection{Passivformen der intransitiven Verben haben keinen 'Passivsinn'.}

\subsubsection{Schlüsseloppositionen und -formen}

Vergleichen wir zuerst die kanonischen Unterschiede zur Bildung von PPM unter Berücksichtigung ihrer Passiv- bzw. Aktivbedeutung (prinzipiell dazu Helbig 1976; neuerlich Thieroff 1994).

Abb.11: Transitiva

\begin{tabular}{||l|l|l||}
\hline & Zustandspassiv & Vorgangspassiv \\
\hline [+perfektiv]: herausziehen & + & + \\
\hline [-perfektiv]: schieben & - & + \\
\hline
\end{tabular}

Abb.12: Intransitiva

\begin{tabular}{||l|l|l||}
\hline & Zustands'passiv' & Vorgangs'passiv' \\
\hline [+perfektiv]: ankommen & + & + \\
\hline [-perfektiv]: arbeiten & - & + \\
\hline [-perfektiv]: gehen, laufen & - & + \\
\hline
\end{tabular}


Abb. 13: unpersönliche 'Passive'

\begin{tabular}{||l|l|l||}
\hline & Zustands`passiv' & Vorgangs'passiv' \\
\hline [+perfektiv]: ankommen $\left(=\mathrm{eVV}^{\prime}\right)$ & - & $-(?)$ \\
\hline [-perfektiv]: arbeiten & - & + \\
\hline [-perfektiv]: gehen, laufen & - & + \\
\hline
\end{tabular}

Das Fragezeichen in der Spalte für 'VoPassiv' und '[+perf]' bezieht sich auf Beispiele wie $A b$ jetzt wird angekommen, etwa in einer Regieanweisung vom Regisseur auf der Bühne - was ja durchaus plausibel erscheint. Vgl. (35).

(35)a es/*er wird/ wurde gelaufen [-passiv]

b detransitives Passiv: er wird geschlagen [+passiv]

c es wird geschlagen [-passiv]

Wir wollen sagen, das unpersönliche morphologische Passiv habe deswegen keinen Passivsinn, weil das Subjekt keine thematische Rolle trägt. Aber dies hat sich als Epiphänomen erwiesen: Passivsinn stellt sich deshalb nicht ein, weil bei den imperfektiven Verben (gleichgültig ob iV oder $\mathrm{tV}$ ) keine telisierende Argumente (Satzglieder/Valenzen) vorliegen. Vgl. den Unterschied zwischen (36a) und (36b).

(36)a *E $r$ wird gelaufen und Es wird gelaufen

b Er/Es wird geschlagen

\subsection{Voraussetzung für PPM: Zustandhaftigkeit $(=[+$ perfekt $],[\alpha$ passiv $])$}

Wie kommt Passiv im Deutschen überhaupt zustande? Was könnten eindeutige Nachweise dafür sein, daß das deutsche Passiv eventuell ein Epiphänomen ist - d.h. daß es aus bestimmten Aspektkonfigurationen abgeleitet ist, dies nicht nur diachron, sondern auch bei synchroner $\mathrm{Be}-$ trachtung? Ich wende mich zuerst der zweiten Frage zu und reihe die Indikationen nach Bedeutsamkeit.\}

\subsubsection{Unpersönliches 'Passiv'}

Das sog. 'unpersönliche Passiv' ist kein Passiv in dem Sinne, daß keine semantisch-transitive Eigenschaftsübertragung statfindet. Vielmehr wird jeweils ein Progressiv ausgedrückt. Alleine deswegen schon handelt es sich dabei um eine Aspekterscheinung katexochen. Vgl. z.B. den Umstand, daß sich zwischen dem unpersönlichen Aktiv und dem unpersönlichen Passiv an Bedeutung und im Sinne der Ereignisstruktur eines Imperfektivums in (17b) nichts ändert.

(37)a Man tanzt

b Es wird getanzt

c Es wird gehoben

d *Es wird gefunden $/{ }^{\mathrm{K}}$ herausgezogen ...

e ??Es wird gestorben/*versunken
... iV

... abgeleitetes iV

... $\mathrm{tV}$

... perfektives iV $(=$ ' $\mathrm{eV}$ ') 
Dies gilt unabhängig davon, ob das beteiligte Prädikat transitiv oder intransitiv ist, wie (37c) zeigt. Nicht unabhängig dagegen ist diese morphologische Passivform von der AA des beteiligten Prädikats, wie (37d,e) nachweisen. Wir werden allerdings sehen, daß es gilt, einen Unterschied zwischen punktuellen Perfektiven und nicht-punktuellen Perfektiven zu machen. Daraus wird sich auch der Unterschied in (23d-e) ableiten. Hier sei nur so viel dazu gesagt, daß die Inchoativdauerprojektion von wird auf den Ereignispunkt eines punktuellen Perfektivverbs zu keiner plausiblen Bedeutung führt.

Eine der wichtigsten Schlüsselformen ist Es wird gegangen - was ja gerade kein Passiv ist. Vgl. dagegen $E r$ wird gegangen, was so viel heißt wie "Er wird hinausgeschmissen (entlassen)", d.h. wörtlicher "Er wird zum Gehen gebracht". Halten wir vorerst fest: Die unpersönliche Passivform mit dem unthematischen es ist nichtpassivisch, die mit dem thematischen - nämlich patientischen -er dagegen erlaubt fraglos ausschließlich die passivische Interpretation.

Abb. 9 führt zu einer Antwort auf unsere erste Frage oben: "Wie kommt das Passiv im Deutschen zustande?" gegangen wechselt zum passivischen Sinn dann, wenn ein thematisches Subjekt gegeben ist - dann nämlich wird aus dem Zustandsargument - das ja nur ein Subjekt sein kann - die vorpartizipiale Vorphasenform rekonstruiert, in der das Zustandsargument der Nachphase Objekt gewesen sein muß (ähnlich den Gedanken Pustejovskys 1991, ekiensfalls jedoch identisch mit diesen, da dort keine Ereigniskonfiguration zugrundegelegt wurde). Passivischer Sinn ergibt sich also, so können wir jetzt sagen, wenn tatsächlich, wie die Alten schon meinten, ein Handlungstransfer vom Agens zum Endpunkt beim Patiens stattfindet, dann und nur dann. Wo dies deswegen nicht möglich ist, weil keine thematische Rolle den Handlungstransfer übernehmen kann - das Ergebnis dieses Transfers tragen kann - wie bei Intransitiva oder bei unthematischem es in unpersönlichen Konstruktionen, ergibt sich niemals passivischer Sinn, sondern ausschließlich die Vorgangslesart.

\subsubsection{Thematisches und unthematische Passivform}

Auffälligerweise erlaubt ndl. (38d) den Schluß, daß bei Verzicht auf geworden die Präsupposition auf die Vorläuferphase zum PPM gezogen wird - was im Ndl. bereits voll durchgrammatikalisiert ist.

(38)a **er wird gelaufen

b ZuP: *Der Wagen ist geschoben

c nur als Metapher, sonst *:

$\mathrm{d}$ ndl. de kar is geduwd (*geworden) vs. es wird gelaufen

vs. er ist herausgeschoben

Er wird gegangen

Was ist bei (38c) geschehen? Mit dem designierten Objektpatienswird ein telischer Punkt eingeführt. D.h. die Progressivität des unpersönlichen 'Passivs' ist deshalb suspendiert, weil die Situierung des telischen Objekt-NPs das Ereignis quasiperfektiv macht. Es ist zu beachten, daß auch dieser Umstand in direkter Weise für die Aspekthypothese und gegen die Argumenthypothese spricht. 


\subsection{Folgerung}

Aus der Asymmetrie der passivischen Formen wie gezeigt in (4a,b), Abb. 6 und Abb. 7 alleine geht schon hervor, daß zumindest aspektuelle Teilabhängigkeit beim deutschen Passiv vorliegt. Wo Passivsinn nachweisbar wird, muß stets Transitivtät (neben der miteintscheidenden Agentivität des betreffenden Prädikatslexems - wobei Transitivtät immer AGENS voraussetzt) vorliegen. Intransitive Prädikate haben trotz der Passivform niemals Passivsinn (vgl. Abraham 1995: Kap. $3,161 \mathrm{ff}$.). Die Tatsache allerdings, daß sich nun in der Diachronie des Deutschen (und der anderen kontinentalen westgermanischen Sprachen: dem Ndl., dem Westfries. und dem Jiddischen) für eine nichtpassivische Lesart genau die Passivform einpendelte, ist ein klarer Hinweis darauf, daß die aspektuell-temporale Leistung des 2 . Partizips auch synchron als die primäre zu betrachten ist und daß passivischer Sinn sich in Ableitung aus der Zustandsaspektualität des PPM ergibt. Gerade aufgrund der Zustandshaftigkeit des PPM ist Agentivität implizit ausgeschlossen. Somit ist auch Deagentivierung des PPM mit Passsivsinn abgeleitet aus dem Adjektivstatus des PPM.

Im folgenden stelle ich die Kernmechanismen bei Passivsinn im deutschen (und, wie ich zu zeigen bereit bin, in allen Sprachen, die zur Passivbildung PPM verwenden zusammen: z.B. auch im Lateinischen und erst recht im Englischen):

(39) PPM bedeutet Zustandshaftigkeit, damit Adjektivstatus. Dies impliziert seinerseits Agenslosigkeit.

(40) PPM setzt eine Vorphase voraus: und zwar entweder über die Aktionsart bei [+perf] oder über die Tempuskonfiguration bei [-perf] (aber eben nur beim periphrastischen Perfekt, aufgrund der speziellen Vorphasenimplikation des PPM).

(41) Die Passsivsinnentscheidung ist semantisch getragen durch ein telisches Objekt bzw. eine telische Verbpartikel bzw. ein entsprechendes Affixoid; syntaktisch ist er getragen durch die Aux-Selektion wie dargestellt in (27).

Wenn das diachrone Kriterium in (20a,b) stimmt, dann kann (19 E.v.G.) nicht als plausibler Endpunkt des modernen Deutschen nach der Ausgangskonfiguration in (3) herhalten.Die Syntax muß stärker an die Aktionsart- und Tempuskonfiguration in (18)-(19) angelehnt sein.

Es ist nicht uninteressant darauf hinzuweisen, daß sich mit dieser Folgerung auch einiges aber eben doch nicht alles - in der Tradition der typologischen Passivdiskussion an Bedeutsamkeit umrangiert: etwa die vernichtende Kritik, die Nerbonne (1982) an Perlmutters 'One-Advancement-Law' der Arc Pair Grammar (Perlmutter 19\%\%) gerade anhand der deutschen Passive aus Intransitiva lieferte: Da es eben Passive aus Intransitiva wie im Deutschen gebe (die gibt es übrigens auch im passivsynthetischen Lateinischen:itur "es wird gegangen"!), könne Perlmutters Gesetz keine universelle Gültigkeit haben. Es gilt, wie wir gesehen haben, Passivform von passivischem Sinn genau zu unterscheiden. Wenn man dies im dargelegten Sinne tut, dann sind die nur der Form nach 'passivierten' Prädikate im Deutschen keine Gegenbeispiele zu Perlmutters typologischer Voraussage.

\section{Syntax des modernen Englisch}

\subsection{Funktionale Kategorien und Kongruenz}

Im folgenden seien Annahmen aus der Literatur (hier zusammenfassend van Gelderen 1997) zu Strukturen dargestellt, die im Deutschen (und ebenso im Englischen und den anderen europäis- 
chen Sprachen) das PPM enthalten. Diesen Annahmen werden die Folgerungen gegenübergestellt, die im vorliegenden Aufsatz gezogen wurden.

(42) Präsens-Aktivstruktur eine transitiven Satzes (Van Gelderen 1997: 5 - Beispiel (4)):

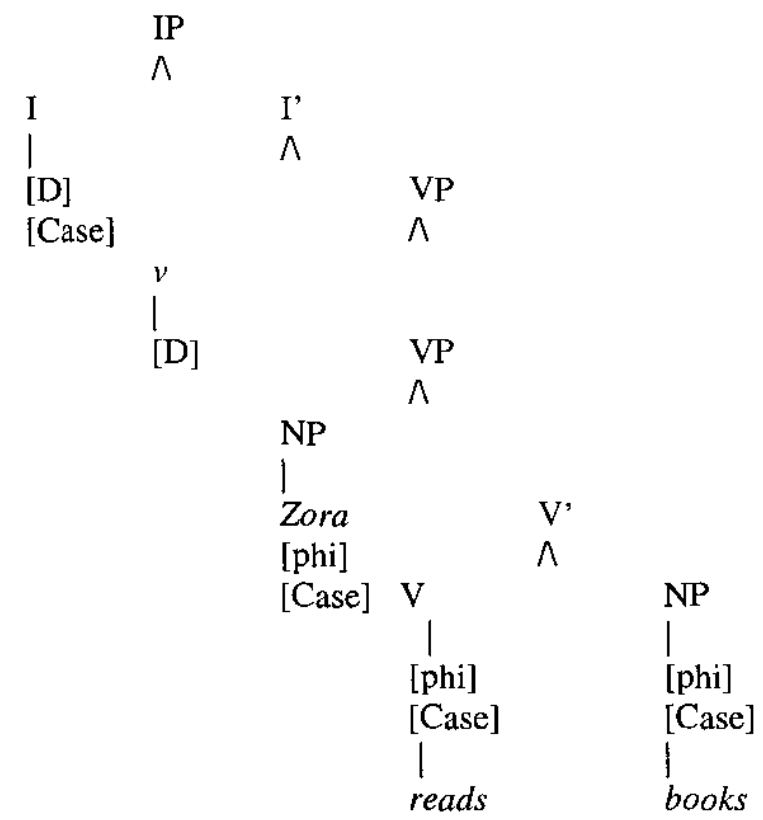

6.2. 'Present perfect' (van Gelderen 1997: 8 - ex. (13))

(43)a

$\mathrm{CP}$

$\wedge$

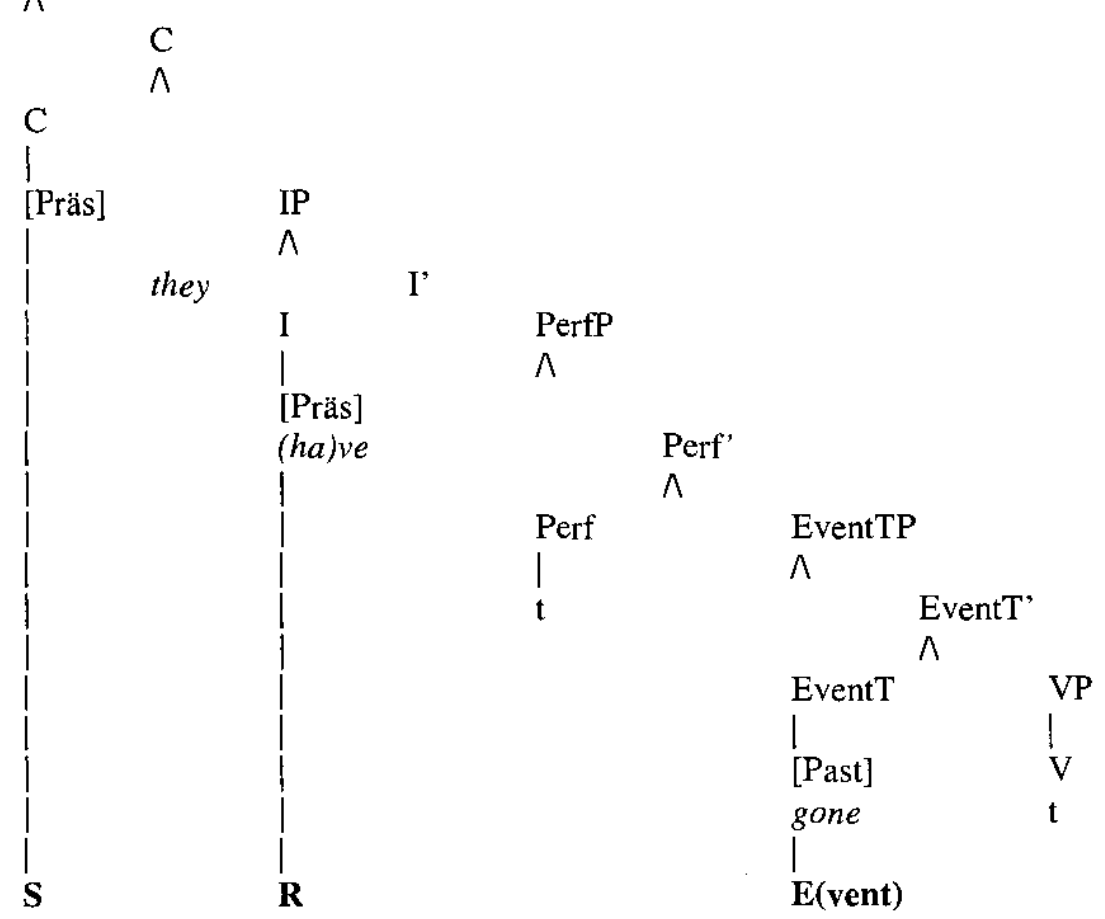


(43)b Ereignisstruktur

$\begin{array}{lll} & \text { Event T } & \\ & \wedge & \\ \text { gone } & & \text { EventT } \\ \text { [Past }] & & \text { [Past] }\end{array}$

"have is taken out of the lexicon as an element with present features that are checked in I; gone has past features and checks those in EventT." (v. Gelderen 1997: 9).

\subsection{Einfaches Präteritum ('Imperfekt') (van Gelderen 1997: 10)}

(44)

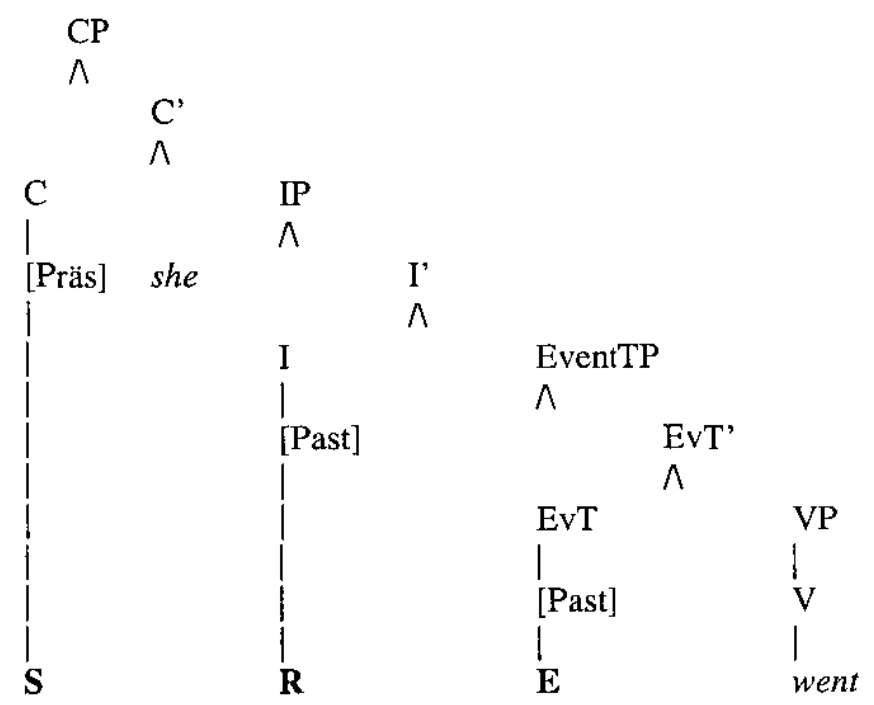

6.4. Progressive+perfect (van Gelderen 1997: 21 (62))

(45)

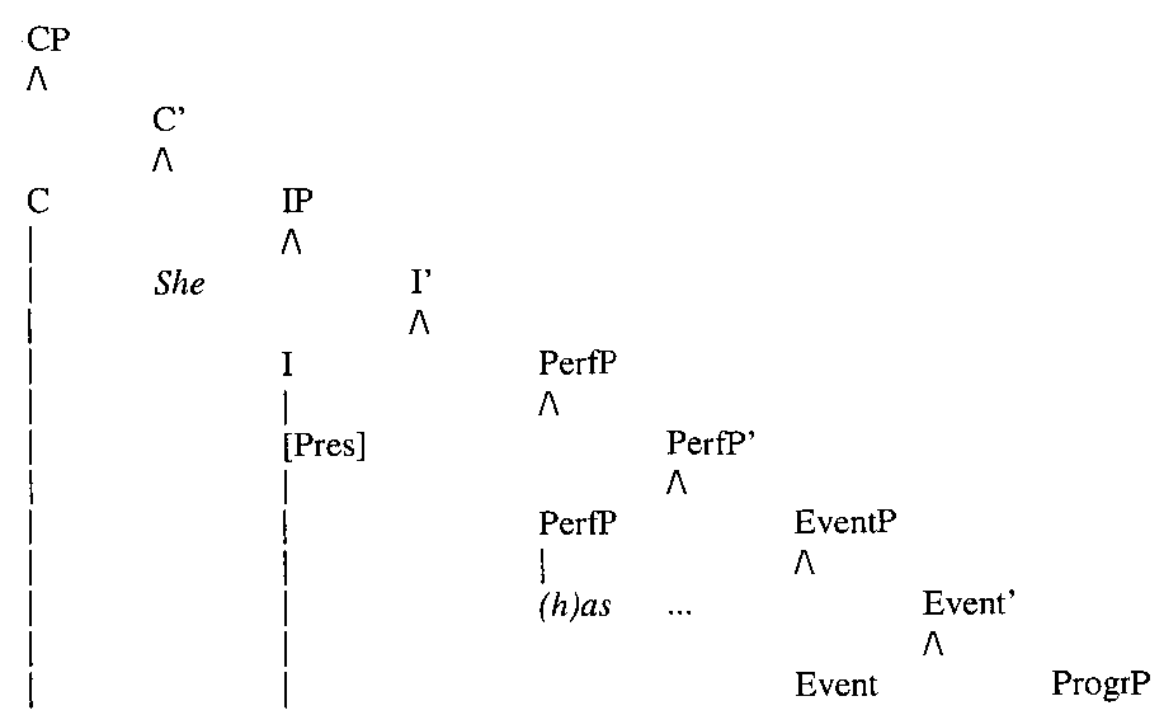




\subsection{Perfective active}

(46) unten gilt nach van Gelderen (1997: 16) für das Aktivperfekt im Englischen, also etwa Someone ate the apple, wobei die telische Präposition unbesetzt bleiben kann!) und wo 'Trans(P)' (= AgrO). Die semantische Kategorie der Telizität sowie das semantische Merkmal [GOAL] entsprechen dem, was öfters, jedenfalls auch in anderen Sprachen, durch eine Verbalpartikel ausgedrückt wird, die betont erscheinen muß (etwa in We received it in for you).

$\wedge$

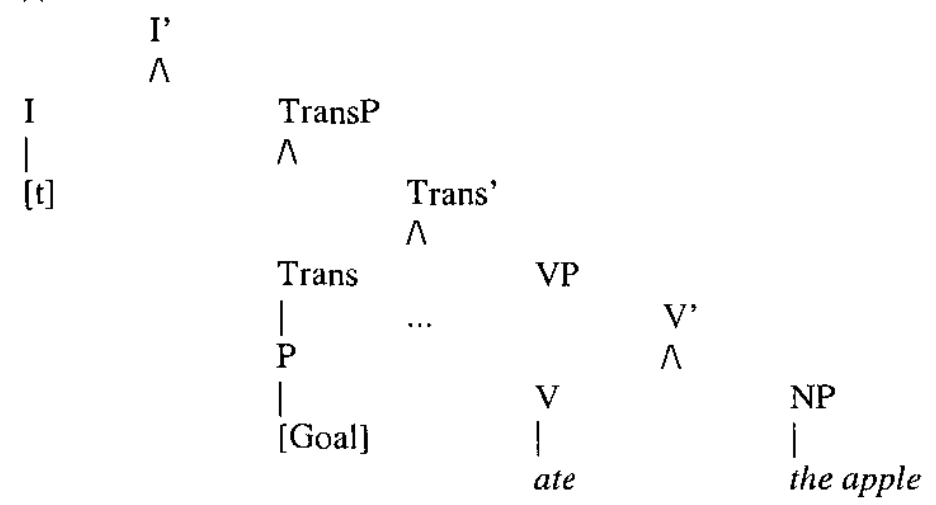

\subsection{Passiv}

(47) gilt (siehe van Gelderen 1997: 23; ihr (68)) für deen Passivsatz She may have been being warned.

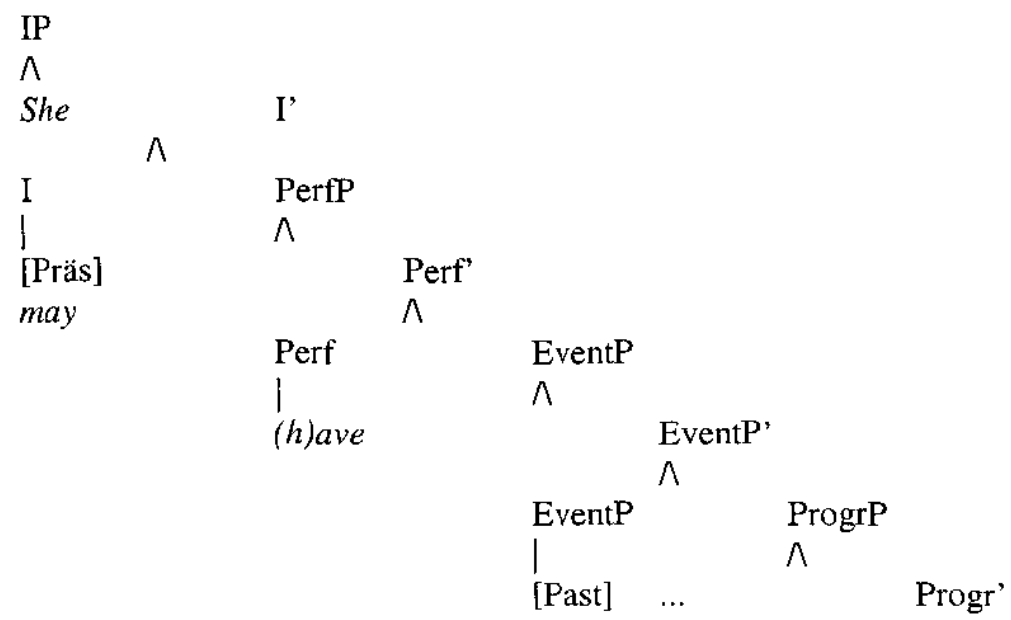




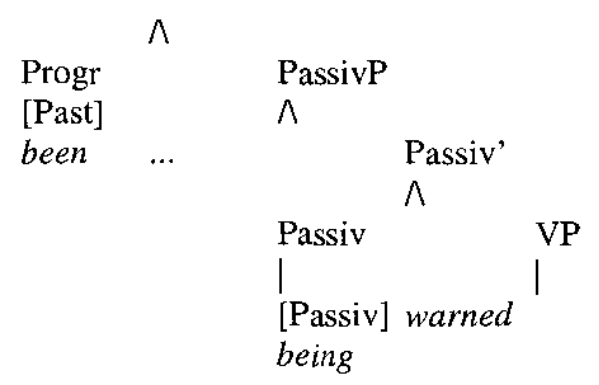

Zu den Strukturargumente, die für (47) sprechen, zählen:

(48)

(a) die Wortstellung ist fest mit der Abfolge: MoDAL-PERFEKT-PROGRESSIV-PASSIV-V

(b) Voranstellung (Topikalisierung) betrifft nur bestimmte Projektionen, andere dagegen nicht (etwa Progressiv, nicht jedoch Perfekt und Passiv)

(c) do so ersetzt VP, aber keine der anderen Projektionen

Um dies erfolgreich diskutieren zu können, werfen wir zuerst einen Blick in die diachrone Entwicklung passivischer Sätze im Deutschen.

\section{Historisch deutliche Verhältnisse: ihre Bedeutung für das Neuhochdeutsche}

\subsection{Semantik}

Im Althochdeutschen und in schwindendem Maße im Mhd. kam Passivisches ausschließlich als Objektprädikation vor: also nur bei Transitiven, wo das Objekt internes Subjekt eines eingebetteten Kurzsatzes (Objektprädikats) und das Gesamtprädikat perfektiv (terminativ) war; internes Prädikat des Kurzsatzes ist dann der perfektivierende Verbteil, meist die Verbpartikel bzw. ein Affixoid. Vgl. (20). ['SQA' = specific quantified argument]

(49)a [+SQA $]_{\mathrm{DO}-\mathrm{TH}}$ im Objektprädikat (Small clause) nur mit TH-Subjekt und verborgener Kopula!) $\Rightarrow$

b $\Rightarrow[" \mathrm{SQA}]_{\mathrm{DO}-\mathrm{TH}}$, somit auch ohne Small clause und allen anderen Folgen; sprich: keine Transitivitätsvorbedingung mehr, keine Perfektivitätsvorbedingung mehr.

(50)a haben: eA-GO[iA-TH-Akk_] ( $\equiv$ sein: eA-TH[iA-GO-Dat_]) $\Rightarrow$ haben:_[v__]

b werden kommt hinzu: zuerst als inchoatives Vollverb mit der Selektion $[ \pm N, \mp \mathrm{V}]$, also ausschließlich Adjektivischen und Nominalen, später stark eingeschränkt auch [-N,+V], also Verbformen.

\subsection{Syntax}

Die umfassende, ausnahmslose Beschränkung auf [+SQA] beim passivierten finiten Prädikat wurde auf dem Weg zum Neuhochdeutschen aufgegeben. Daraus folgt allerdings nicht, daß die SC-Syntax insgesamt aufgegeben wurde, sondern bloß daß eine solche ursprünglich an [+SQA] gebundene $\mathrm{SC}(=$ Objektprädikat $)-S y n t a x$ in eingeschränkterem Maße gilt. Weiter entwickeln sich haben und werden zu 'Hilfsverben', d.h. zu Hebungsverben ohne eigene thematische Selektion für Subjekt und Objekt. Vgl. weiter unten. 
Zur Struktur des Verbs im Deutschen sei im Gegensatz zu den Annahmen fürs Englische oben folgende Strukturannahme getroffen.

$$
\mathrm{VP}
$$

$\wedge$

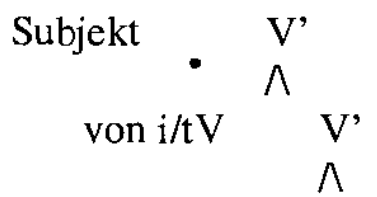

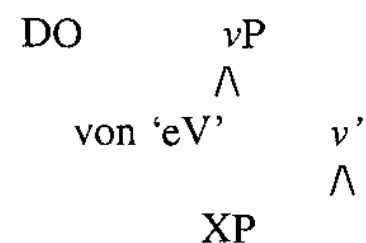

$\{$ in den Graben $\}$

\{hinein $\}$

\{das Haus\}

$\mathrm{XP}$ unter $v^{\prime}$ ist das interne Argument von $v$. Vergleichen wir mit (3) oben, der Struktur des althochdt. Objektprädikats.

(52) Er hat den Baum gepflanzt

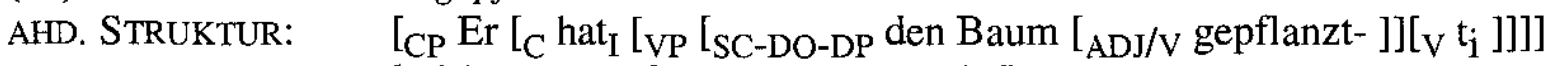

habêt boum gipflanzotan

= SPÄTLAT. STR.: habet arborem plantatum

NHD. STRUKTUR: [ ${ }_{C P} \operatorname{Er}_{C_{C}}$ hat $_{I}\left[_{V P}\left[{ }_{D O-D P}\right.\right.$ den Baum] [V gepflanzt $\left.\left.\left.t_{i}\right]\right]\right]$

${ }_{\mathrm{CP}} \operatorname{Er}\left[\mathrm{C}\right.$ bekam $_{\mathrm{I}}\left[_{\mathrm{VP}}[\mathrm{SC}-\mathrm{DO}-\mathrm{DP}\right.$ den Baum $[\mathrm{ADJ} / \mathrm{V}$ schon gepflanzt $\left.\left.]]\left[\mathrm{V}_{\mathrm{i}}\right]\right]\right]$ $\cong$ Er bekam den Baum (als) gepflanzten

(53) Struktur des ahd. Satzes sowie des nhd. bekommen-Satzes:

IP

$\Lambda$

er

$\wedge$

VP

$\wedge$

$\mathrm{t} \quad \mathrm{V}^{\prime}$

$\wedge$

den Baum $\quad v \mathrm{P}$

$\wedge$

$\mathrm{t} \quad v^{\prime}$

$\mathrm{XP} \quad \wedge$

geplanzt(-) hat/bekommt 
Die Passivstruktur im Ahd. ist dann die folgende.

(54) Passivstruktur im Ahd.

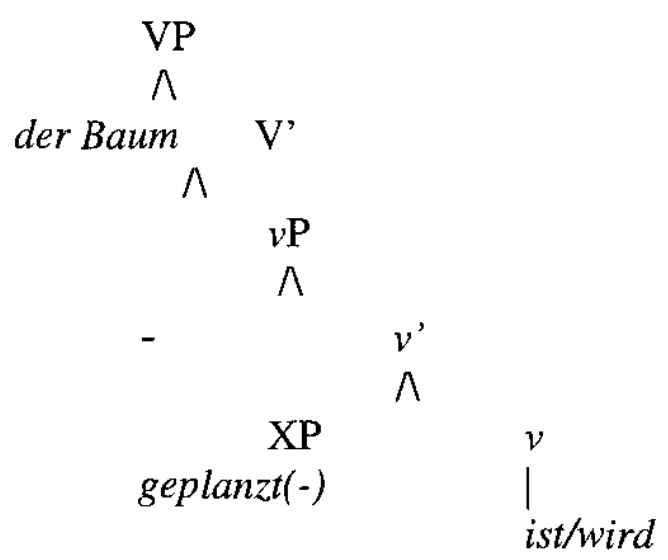

Die Satzstruktur in (54) ist unvollständig insoferne, als ein entsprechender funktionaler Knoten für die Kongruenzmoprphologie am PPM zu sorgen hat. Dieser Kongruenzknoten fehlt in (6).

(55) Passivstruktur im Nhd.: entstanden durch Strukturvereinfachung (es gibt kein $v \mathrm{P}$ mehr); der funktionale Kongruenzknoten für das ahd. Objektprädikat fehlt im Nhd..

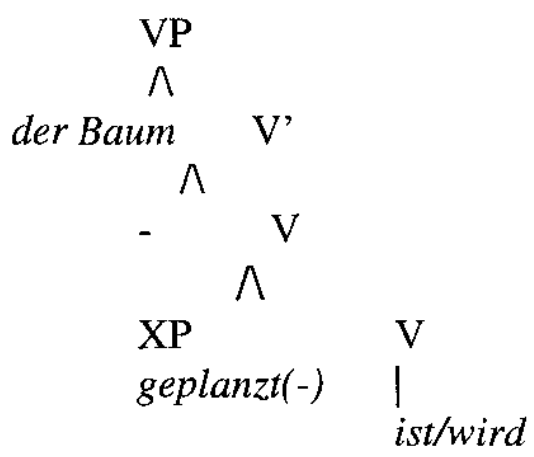

Die diachrone Entwicklung von (5) zu (6) trägt dem empirischen Befund Rechnung, daß es keine eigene passivartige Verbklasse der ' $\mathrm{eV}$ ' gibt. Der Umstand, daß das periphrastische Prädikat dennoch Ähnlichkeit mit der Struktur des Ahd. (und Russischen) aufweist, zeigt sich darin, daß der Komplementcharakter des PPM in Abhängigkeit von AUX identisch dargestellt wird.

Unter den bisher entwickelten Annahmen hat sich die Passivstruktur im Neuhochdeutschen gegenüber dem Althochdeutschen nicht geändert: das PPM denotiert über Implikatur die Vorgängerphase. Die entscheidende Änderung ist die Selektionseinschränkung bei werden: dieses beschränkt seine inchoative, lexikalische volle Verbbedeutung auf die Adjektivkategorie und damit das PPM (unter Einschluß des Präsenspartizips, dies jedoch unter hoher stilistischer Markierung). Bei der Selektion von V nimmt werden eindeutig Hilfsverbcharakter an. 


\section{Schluß}

Es spricht für den aus seiner diachronen Einsicht genährten Spürsinn Behaghels (1924) für das moderne Deutsch, daß er den hier getroffenen Anschluß des alten (des ahd.) zum neuen (zum nhd.) Passiv ermöglicht - ohne dies freilich explizit auszusprechen. Man vgl. die folgenden $\mathrm{Zi}$ tatausschnitte: "Ein persönliches Passiv bilden Verba, die einen Akk. Zu sich nehmen, falls diese eine zu einem Ergebnis herbeiführende Tätigkeit bezeichnen" (Behaghel 1924: 211). Und weiter: "Ein unpersönliches Passiv bilden Verba mit Ergänzung durch Gen. Oder Dativ oder ohne kasuelle Ergänzung, die eine Tätigkeit bezeichnen" (ebd. 211). Die allgemeinen Bedingungen der Passivfähigkeit stellt Behaghel folgendermaßen dar: "A. Daß dabei das Zeitwort werden beteiligt ist, das einen Vorgang, eine Veränderung bezeichnet. B. Daß dabei das Part.Prät. beteiligt ist, das ursprünglich ein Adjektiv ist und den durch eine Tätigkeit geschaffenen Zustand bezeichnet. C. Daß die Bezeichnung des Urhebers der Handlung beim Passiv ursprünglich keine stelle hat." (Behaghel 1924: 210f.). Dies spricht gegen die weitaus allgemeineren und mit den diachronen Verhältnissen des Germanischen unvereinbaren Definitionskomponenten Shibatanis (1985) in (2) oben. Q.E.D.

\section{Addendum}

Zu den wesentlichen Zusammenhängen, die ich für Aspekt und Verbdiathese skizziert habe, gehören auch die folgenden, die zwischen den Hilfsverben im Deutschen bestehen und die die vorgestellten Beschreibungen erklärlicher machen.

Komposition der Hilfsverben:

(56) $w \operatorname{erden}(\mathrm{e})$

$$
\begin{aligned}
& \Leftrightarrow \quad e\left(e_{1}, e_{2}\right) \wedge \operatorname{INCHOATIV}\left(e_{1}\right) \wedge\left[R_{\text {RS }} \operatorname{ZUSTAND}\left(\mathrm{e}_{2}\right) \wedge \operatorname{Culm}\left(\mathrm{e}_{1}\right) \wedge\right. \\
& \left.\mathrm{e}\left(\mathrm{e}_{1}<\mathrm{e}_{2}\right)\right]
\end{aligned}
$$

(57) haben/eignen $\left(\mathrm{e}_{2}\right) \Leftrightarrow \quad \operatorname{ZuSTAND}\left(\mathrm{e}_{2}\right) \wedge \mathrm{e}_{2}(\mathrm{x}, \mathrm{y}) \wedge \operatorname{POSS}(\mathrm{x}) \wedge \operatorname{TH}(\mathrm{y})$

(58) $\operatorname{sein}\left(\mathrm{e}_{2}\right) \quad \Leftrightarrow \quad \operatorname{ZUSTAND}\left(\mathrm{e}_{2}\right) \wedge \mathrm{e}_{2}(\mathrm{y}) \wedge \mathrm{TH}(\mathrm{y})$

(59) bekommen $(\mathrm{e}) \Leftrightarrow \quad$ haben werden $(\mathrm{e}) \Leftrightarrow\left[\mathrm{e}\left(\mathrm{e}_{1}, \mathrm{e}_{2}\right) \wedge \operatorname{INCHOATIV}\left(\mathrm{e}_{1}\right) \wedge \operatorname{Culm}\left(\mathrm{e}_{1}\right) \wedge\right.$ $\mathrm{e}\left(\mathrm{e}_{1}<\mathrm{e}_{2}\right) \wedge$ haben $\left.(\mathrm{e})\right]$

Es gibt somit lexikalische Zusammenhänge: zwischen haben und sein einerseits, vergleichbar etwa dem klasischen Lateinischen und Spätlateinischen, wo habere(x-Nom,y-Akkusativ) einem esse( $\mathrm{y}$-Dativ, $\mathrm{x}$-Nominativ) entsprach; zwischen bekommen (wesentlich für das Dativpassiv) und haben werden; sowie zwischen haben und werden bzw. sein insoferne, als die Verteilung der Ereignisunterabschnitte sich spezifisch auf die drei Prädikate verteilt.

\section{Bibliographie}

Abraham, Werner 1987. "Burzio trifft Wulfila. Zu den distributionellen Eigenschaften von wairpan "werden" und wisan "sein" im gotischen Passiv." Groningen Papers in Theoretical and Applied Linguistics - TTT 9: 1-29. 
Abraham, Werner 1993 "The aspect-case typology correlation: perfectivity triggering split ergativity." Folia Linguistica XXX/1-2: 5-34.

Abraham, Werner 1998a. "The loss of the preterite in the European languages." Folia Linguistica Europaea (erscheint 1999).

Abraham, Werner 1998b. "Der angebliche Passivstatus des Perfektpartizips." Groninger Arbeiten zur germanistischen Linguistik 42.

Abraham, Werner \& Wladimir Klimonow 1999. "Typologisch-kontrastive Miszellen: Perfektivität ubiquiter - Ergativität nusquam." In: H. Wegener (hg.) Deutsch kontrastiv. Tübingen: Stauffenburg, 1-32.

Andersson, Sven-Gunnar "Typologisches und Funktionales beim Passiv und Reflexiv im Deutschen, Schwedischen und Russischen." SLE St. Andrews 1998 handout.

Beedham, Christopher 1981. "The passive in English, German, and Russian." Journal of Linguistics 17, 319-327.

Beedham, Christopher 1998. "The perfect passive participle in Russian: A review of Participial passive and aspect in Russian von Maaike Schoorlemmer 1995. Review article Lingua 105, 7994.

Behaghel, Otto 1924. Deutsche Syntax. Eine geschichtliche Darstellung. Band II: Die Wortklassen und Wortformen. Heidelberg: C. Winter.

Comrie, Bernard 1985 Tense. Cambridge: CUP.

Dowty, David R. 1979 Word meaning and Montague grammar. The semantics of verbs and times in Generative Semantics and in Montaque's PTQ. Dordrecht: Reidel

Gelderen, Elly van 1997. "Structures of tense and aspect". Linguistic Analysis 27:3-4, 1-28.

Giorgi, Alessandra \& Fabio Pianesi 1997. Tense and aspect. From semantics to morphosyntax. Oxford: OUP.

Helbig, Gerhard \& J. Buscha 1976. Deutsche Übungsgrammatik. Leipzig: VEB.

Hornstein, Norbert 1990. As time goes by. Cambridge, Massachusetts: MIT Press,

Mahajan, Anoop 1994. Universal grammar and the typology of ergative languages. Paper UCLA. Read at the FAS-meeting Berlin, November 1994.

Parson, Terence 1990. Events in the semantivcs of English. Cambridge, Massachusetts: MIT Press. 
Maslow, J.S. 1957. "Glagol'nij vid v sovremennom literaturnom bolgarskom jazyke; značenije i upotreblenije." In: S.B. Bernštejn (hg.) Voprosy grammatiki sovremennogo literaturnogo bolgraskogo jazyka. Moskau: Nauka, 157-312.

Nerbonne, John 1982. 'Some passives not characterized by universal; rules: subjectless impersonals.' In: Brian Joseph (ed) Relational Grammar and grammatical relations. OSU Working Papers in Linguistics 26: 59-92.

Pustejovsky, John 1991. 'The syntax of event structure'. Cognition 41:47-81. (ebenso in Beth' Levin \& Stephen Pinker 1992 (hg.) Lexical and Conceptual Semantics. Oxford: Blackwell. (=Wiederabdruck des Cognition-Aufsatzes).

Schoorlemmer, Maaike 1995. Participial passive and aspect in Russian. Dissertation OTS-Univ. Utrecht 1995.

Thieroff, Rolf 1994. 'Vorgangs- und Zustandspassiv in romanischen und germanischen Sprachen.' Sprachtypologische Universalforschung (STUF) 47-1, 37-54. 